\title{
Chandra X-ray spectroscopy of the focused wind in the Cygnus X-1 system
}

\section{Dipping in the low/hard state}

\author{
Maria Hirsch ${ }^{1}$, Natalie Hell ${ }^{2}$, Victoria Grinberg ${ }^{3}$, Ralf Ballhausen ${ }^{1}$, Michael A. Nowak ${ }^{4}$, Katja Pottschmidt ${ }^{5,6}$, \\ Norbert S. Schulz ${ }^{7}$, Thomas Dauser ${ }^{1}$, Manfred Hanke ${ }^{1}$, Timothy R. Kallman ${ }^{6}$, Gregory V. Brown ${ }^{2}$, and Jörn Wilms ${ }^{1}$ \\ ${ }^{1}$ Dr. Karl Remeis-Sternwarte and Erlangen Centre for Astroparticle Physics, Universität Erlangen-Nürnberg, Sternwartstr. 7, \\ 96049 Bamberg, Germany \\ e-mail: maria.hirsch@sternwarte.uni-erlangen.de \\ ${ }^{2}$ Lawrence Livermore National Laboratory, 7000 East Ave., Livermore, CA 94550, USA \\ ${ }^{3}$ Institut für Astronomie und Astrophysik, Universität Tübingen, Sand 1, 72076 Tübingen, Germany \\ ${ }^{4}$ Department of Physics, Washington University in St. Louis, Campus Box 1105, One Brookings Drive, St. Louis, \\ MO 63130-4899, USA \\ ${ }^{5}$ CRESST, Department of Physics, and Center for Space Science and Technology, UMBC, Baltimore, MD 21250, USA \\ ${ }^{6}$ NASA Goddard Spaceflight Center, 8800 Greenbelt Rd, Greenbelt, MD 20771, USA \\ ${ }^{7}$ MIT Kavli Institute for Astrophysics and Space Research, NE80-6077, 77 Mass. Ave., Cambridge, MA 02139, USA
}

Received 17 January 2019 / Accepted 22 April 2019

\begin{abstract}
We present an analysis of three Chandra High Energy Transmission Gratings observations of the black hole binary Cyg X-1/HDE 226868 at different orbital phases. The stellar wind that is powering the accretion in this system is characterized by temperature and density inhomogeneities including structures, or "clumps", of colder, more dense material embedded in the photoionized gas. As these clumps pass our line of sight, absorption dips appear in the light curve. We characterize the properties of the clumps through spectral changes during various dip stages. Comparing the silicon and sulfur absorption line regions $(1.6-2.7 \mathrm{keV} \equiv 7.7-4.6 \AA)$ in four levels of varying column depth reveals the presence of lower ionization stages, i.e., colder or denser material, in the deeper dip phases. The Doppler velocities of the lines are roughly consistent within each observation, varying with the respective orbital phase. This is consistent with the picture of a structure that consists of differently ionized material, in which shells of material facing the black hole shield the inner and back shells from the ionizing radiation. The variation of the Doppler velocities compared to a toy model of the stellar wind, however, does not allow us to pin down an exact location of the clump region in the system. This result, as well as the asymmetric shape of the observed lines, point at a picture of a complex wind structure.
\end{abstract}

Key words. accretion, accretion disks - stars: individual: HDE 226868 - stars: individual: Cyg X-1 - stars: winds, outflows techniques: spectroscopic - X-rays: binaries

\section{Introduction}

Discovered during a balloon flight in 1964 (Bowyer et al. 1965), Cygnus X-1 is one of the best studied black hole X-ray binaries. Based on radio parallax data, the distance of the system was measured to be at $1.86_{-0.11}^{+0.12} \mathrm{kpc}$ (Xiang et al. 2011; Reid et al. 2011) ${ }^{1}$. Cyg X-1 consists of a $(14.8 \pm 1.0) M_{\odot}$ black hole that accretes from the strong stellar wind of the $(19.2 \pm 1.9) M_{\odot}$ supergiant O9.7 Iab star HDE 226868 (Murdin \& Webster 1971; Webster \& Murdin 1972; Walborn 1973; Herrero et al. 1995; Caballero-Nieves et al. 2009; Orosz et al. 2011). The star and the black hole are in a quasi-circular (eccentricity $e=0.018 \pm 0.002$; Orosz et al. 2011) 5.599829(16) d orbit (Webster \& Murdin 1972; Brocksopp et al. 1999; Gies et al. 2003) with an inclination of $i=27.1^{\circ} \pm 0.8^{\circ}$ (Orosz et al. 2011). This corresponds to a separation between the center of mass of the star and the black

1 New Gaia measurements appear to favor a slightly larger distance of $2.38_{-0.17}^{+0.20} \mathrm{kpc}$, possibly due to systematic error caused by the high optical brightness of the system and/or an orbital wobble (Gandhi et al. 2019). hole of only $42.2 R_{\odot}$, or 2.5 stellar radii. Combined with the high mass-loss rate of HDE $226868\left(\sim 10^{-6} M_{\odot} \mathrm{yr}^{-1}\right.$; Puls et al. 2006; Herrero et al. 1995), this small separation means that the black hole is continuously accreting material from the stellar wind, making Cyg X-1 one of the few persistent black holes in our Galaxy.

With its high equivalent hydrogen column density, $N_{\mathrm{H}}$, of around $6 \times 10^{21} \mathrm{~cm}^{-2}$ (Dotani et al. 1997; Schulz et al. 2002a; Miller et al. 2002; Hanke et al. 2008), Cyg X-1 is associated with an X-ray dust scattering halo (see Bode et al. 1985, for the first analysis of this halo). Xiang et al. (2011) found a dust containing cloud in the interstellar medium (ISM) at $\sim 0.885 D$, where $D$ is the distance of the system, to be the region responsible for the scattering halo.

Already soon after the identification of the optical counterpart of Cyg X-1, X-ray light curves were found to show a strong orbital modulation of the X-ray absorption column, $N_{\mathrm{H}}$, due to absorption of X-rays from the black hole in the stellar wind (Li \& Clark 1974; Remillard \& Canizares 1984; Bałucińska-Church et al. 2000; Poutanen et al. 2008; Miškovičová et al. 2016; 
Grinberg et al. 2015). Together with observations of the orbital modulation of optical lines from HDE 226868 (Gies \& Bolton 1986a,b; Gies et al. 2003), these phenomena led to the picture of the stellar wind of HDE 226868 as a line driven wind or CAK wind after Castor et al. (1975, see also Friend \& Castor 1982 and Morton 1967) with an asymptotic velocity of $v_{\infty} \sim 2000 \mathrm{~km} \mathrm{~s}^{-1}$ (Muijres et al. 2012). This wind is disturbed by the gravitational potential of the black hole, which leads to a focusing of the wind toward the black hole; see Friend \& Castor (1982) and Gies \& Bolton (1986b) for early models and El Mellah et al. (2019) for a modern treatment of this process. In addition, the wind is also affected by the X-rays from the compact object: strong orbital modulation of $N_{\mathrm{H}}$ is seen during the canonical hard state of the black hole, where the X-ray spectrum is dominated by a Comptonized power law (Parker et al. 2015; Nowak et al. 2011; Wilms et al. 2006, and references therein). The typical bolometric luminosity of Cyg X-1 in this state is around $2 \times 10^{37} \mathrm{erg} \mathrm{s}^{-1}$ (e.g., Wilms et al. 2006; Nowak et al. 1999), albeit with a large uncertainty due to our lack of knowledge of the UV spectral shape. Only very little $N_{\mathrm{H}}$ modulation is seen during the thermally dominated X-ray soft state (Wen et al. 1999; Boroson \& Vrtilek 2010), in which the typical bolometric luminosity is at most a factor two higher than in the hard state (e.g., Tomsick et al. 2014; Zhang et al. 1997). Optical spectra show the stellar wind to be strongly photoionized during the latter state (Gies et al. 2003, 2008).

Line driven winds are not expected to be smooth flows, but show strong density perturbations or "clumps" (Owocki et al. 1988; Feldmeier et al. 1997; Puls et al. 2006, 2008; Oskinova et al. 2012; Sundqvist \& Owocki 2013). In X-ray binaries, the density contrast could even be further enhanced by the interaction between the wind and the strong X-rays from the compact object (Blondin 1994; Blondin \& Woo 1995; Manousakis \& Walter 2011, 2015, and references therein). For Vela X-1 and Cyg X-1 it has been estimated that more than $90 \%$ of the wind mass is contained in less than $10 \%$ of the wind volume (Sako et al. 1999; Rahoui et al. 2011). When the line of sight to the compact object passes through one of these clumps, X-rays are absorbed by the moderately ionized material in the clump, leading to a so-called dipping event. This is also observed for other sources (Hemphill et al. 2014; Grinberg et al. 2017). During the hard state of Cyg X-1, such short-term dipping events are observed predominantly during the upper conjunction of the black hole, i.e., when the line of sight passes through the densest region of the stellar wind and is most likely to pass through a clump (Li \& Clark 1974; Mason et al. 1974; Parsignault et al. 1976; Pravdo et al. 1980; Remillard \& Canizares 1984; Kitamoto et al. 1984; Bałucińska-Church et al. 2000; Feng \& Cui 2002; Poutanen et al. 2008; Hanke et al. 2009; Miškovičová et al. 2016; Grinberg et al. 2015). The precise structure of the clumps, i.e., their density and ionization structure, is unknown. Most recent 2D simulations of such a stellar wind show a very complex evolution of velocity and density structures with the formation of characteristic small-scale clumps of various shapes embedded in areas with lower density (Sundqvist et al. 2018). Sundqvist et al. (2018) have found a typical clump mass of $10^{17} \mathrm{~g}$ and an average clump size of $1 \%$ of the stellar radius at a distance of two stellar radii. These results qualitatively confirm earlier theoretical models (e.g., Oskinova et al. 2012; Sundqvist \& Owocki 2013) and observations (e.g., Grinberg et al. 2015). See also the review paper by Martínez-Núñez et al. (2017).

In this paper we present the first detailed high-resolution study of absorption dips in Cyg X-1 using time-resolved X-ray spectroscopy during dips observed with the High Energy Transmission Grating Spectrometer (HETGS) on board Chandra. The analysis of a series of Chandra-HETGS observations of the source taken during the low/hard state allows us to investigate the dipping mechanism and to probe the ionization state of the absorber directly. This is the third paper in a series devoted to a study of Cyg X-1 using Chandra high-resolution spectroscopy. Our previous analyses addressed the stellar wind in Cyg X-1 in the hard state as seen outside the absorption dips, i.e., we analyzed the hot tenuous phase of the wind. In the first paper of the series we investigated the wind at $\phi_{\text {orb }} \sim 0$ (Hanke et al. 2009, hereafter Paper I). We extended this work to the orbital modulation of the wind using non-dip observations at $\phi_{\text {orb }} \sim 0.0, \sim 0.2$, $\sim 0.5$, and $\sim 0.75$ (Miškovičová et al. 2016, hereafter Paper II). These papers provide us with a reference for the "normal" (non-dip) hard-state spectrum of Cyg X-1.

In this paper, we address the spectral changes during dipping episodes. We discuss our data reduction and identification of the dipping events in Sect. 2. In Sect. 3 we study the spectral evolution of the silicon and sulfur line regions from the non-dip to the deepest dip and we show that absorption lines of lower ionized species appear as the line of sight crosses through denser regions of the absorbing clump. During the deepest dips the line of sight is fully blocked, revealing line emission from the photoionized plasma around the black hole. We summarize our results in Sect. 4.

\section{Data reduction}

Out of the Chandra-HETGS observations discussed in Paper II for their non-dip properties, we selected a subsample of three observations (Table 1), namely ObsIDs 3814, 8525, and 9847, which show distinct dipping episodes in their light curves (Fig. 1, Sect. 2.1). As discussed in Paper II, the other ObsIDs available were taken in the intermediate and soft states and thus the material in the system was in a different radiative environment. All observations selected for further analysis of the dips were performed in timed exposure (TE) mode. In this mode the usual frame time is $3.2 \mathrm{~s}$ exposure before the data are transferred into a frame store for readout. Cyg X-1 is a bright source even in the low/hard state. Therefore, for the discussed observations only a half array of the chips (512 CCD rows) was read out to minimize pileup by reducing the frame time to $1.7 \mathrm{~s}$. We re-extracted the data using the Chandra Interactive Analysis of Observations (CIAO) software version 4.6 with all parameters set to default except for the HETG cross dispersion extraction width (width_factor_hetg parameter in CIAO), which we reduced from 35 to 10 for better coverage at the short wavelength end.

For the light curves and spectral analysis we used the first order spectra of the high and medium energy gratings (HEG, MEG; Canizares et al. 2005). Because of the excellent background discrimination of the data extraction process (order sorting), any remaining background is negligible compared to the bright source. We therefore did not subtract any background from the final spectra. All further data analysis was performed with the Interactive Spectral Interpretation System version 1.6.2 (ISIS; Houck \& Denicola 2000; Houck 2002; Noble \& Nowak 2008).

\subsection{Light curves}

The light curves of ObsIDs $3814(\sim 48 \mathrm{ks})$ and 8525 $(\sim 30 \mathrm{ks})$ slightly overlap in orbital phase $\left(\phi_{\text {orb }}=0.93-0.03\right.$ and $\left.\phi_{\text {orb }}=0.02-0.08\right)$ and show a very similar morphology of strong absorption dips. ObsID $9847(\sim 19 \mathrm{ks})$ at $\phi_{\text {orb }}=0.17-0.21$ was taken within the same binary orbit as ObsID 8525; it contains 


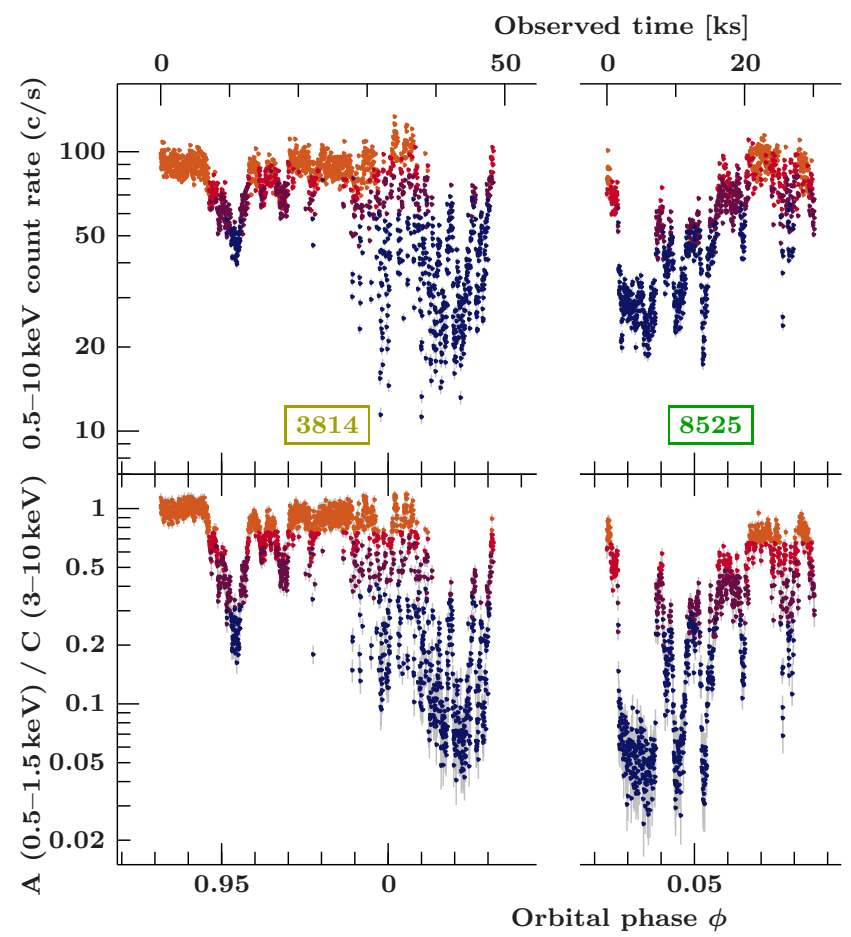

Table 1. Chandra-HETGS observations used in this paper.

\begin{tabular}{llllll}
\hline \hline \multirow{2}{*}{ ObsID } & \multicolumn{2}{c}{ Start date } & & & \\
\cline { 2 - 3 } & $\begin{array}{l}\text { Date } \\
\text { yyyy-mm-dd }\end{array}$ & MJD & Mode & $\begin{array}{l}T_{\text {exp }} \\
(\mathrm{ks})\end{array}$ & $\phi_{\text {orb }}$ \\
\hline 3814 & $2003-04-19$ & 52748 & TE/g & 48.3 & $0.93-0.03$ \\
8525 & $2008-04-18$ & 54574 & TE/g & 30.1 & $0.02-0.08$ \\
9847 & $2008-04-19$ & 54575 & TE/g & 19.3 & $0.17-0.21$ \\
\hline
\end{tabular}

Notes. TE/g: timed exposure, graded; $T_{\text {exp }}$ : exposure time; $\Phi_{\text {orb }}$ : orbital phase according to the ephemeris of Gies et al. (2003).

absorption dips as well, although they are less pronounced than at $\phi_{\text {orb }} \sim 0$. Dips can last from several seconds to more than 10 min (Kitamoto et al. 1984). We extracted light curves with a $25.5 \mathrm{~s}$ resolution to uncover the shorter dips as well. For a detailed description of the data set and a detailed analysis of the non-dip spectrum of individual observations, we refer to Papers I and II. Paper II also discusses ObsID $11044(\sim 30 \mathrm{ks})$, which at $\phi_{\text {orb }}=0.48-0.54$ for the first time provides high-resolution spectroscopy of Cyg X-1 during lower conjunction, i.e., when our line of sight passes outside of the focused wind. As this light curve appears to be virtually free of dipping, we do not discuss this light curve in detail in this work. Cyg X-1 was in a comparable hard state during ObsIDs 3814, 8525, and 9847 according to the Rossi X-ray Timing Explorer (RXTE) All Sky Monitor classification by Grinberg et al. (2013); see also the more detailed discussion of the source state in Paper II. We do not use ObsID 3815 as it shows much less dipping than the other hard state observations, which does not allow for a distinction between different dip stages (Paper II, see also Miškovičová et al. 2011).

\subsection{Color-color diagrams}

As we discuss in more detail below, the dips are due to absorption events caused by material in the line of sight to the primary

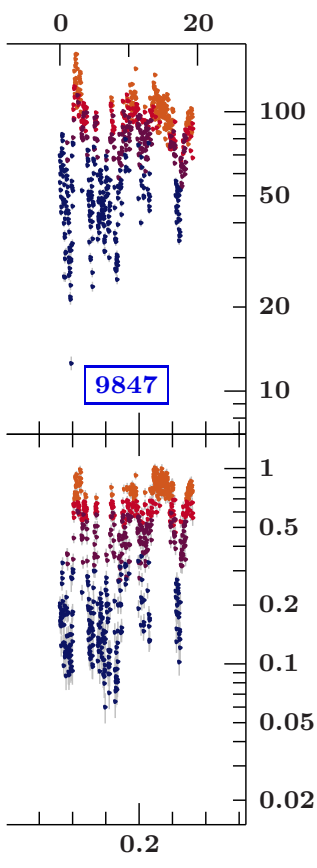

Fig. 1. Top panel: light curves with a $25.5 \mathrm{~s}$ time resolution of all three observations as a function of orbital phase. Bottom panel: corresponding hardness ratios. All observations were in the low/hard state. Dips are strongest at $\phi_{\text {orb }} \sim 0.0$, become weaker at $\phi_{\text {orb }} \sim 0.2$ and $\phi_{\text {orb }} \sim 0.75$, and completely fade at $\phi_{\text {orb }} \sim 0.5$ (see also Paper II). Colors indicate the selected dipping stages (see right panel of Fig. 3 for the color denotation and Sect. 2.3 for details on the data selection). source of X-rays. Dipping events such as thos seen in Cyg $\mathrm{X}-1$ are transient events characterized by quickly varying spectral shape. This strong variability complicates the spectral analysis. Ideally, we would want to study how the spectral shape - photon index, absorption/emission lines, and continuum absorption varies with time, but the limited signal to noise of our observations renders this impossible. We therefore have to resort to some kind of averaging technique in which we extract spectra from time intervals where we believe that the spectral shape is at least representative for a given part of a dip. We find these time intervals by looking at the time resolved spectral behavior of the source as represented in so-called color-color diagrams.

Figure 2 shows how absorption affects an observed primary power law continuum with $\Gamma=1.73$ (typical for Cyg X-1 in the hard state, e.g., Grinberg et al. 2013) that is fully covered by material with a fixed column density $N_{\mathrm{H}}^{(0)}$ (e.g., the outer parts of the stellar wind or absorption in the ISM). For this paper, we set $N_{\mathrm{H}}^{(0)}$ to the fit result of Paper I, $N_{\mathrm{H}}^{(0)}=5.4 \times 10^{21} \mathrm{~cm}^{-2}$. In most astrophysical sources, the structures responsible for dipping do not cover the whole primary X-ray source, rather a partial coverer is present in these systems that covers a fraction $f_{\mathrm{c}}$ of the source with a column $N_{\mathrm{H}}$ (i.e., $1-f_{\mathrm{c}}$ remains uncovered). A possible physical picture for such a partial coverer would be an optically thick cloud that is smaller in (angular) size than the $\mathrm{X}$-ray source, or a cloud that passes very quickly over the X-ray source, covering the source only for part of the integration time. The observer therefore sees the sum of the uncovered spectrum (dash-dotted line in Fig. 2) and the covered spectrum (dashed lines). The summed spectrum is shown as solid lines in Fig. 2. The left panel of Fig. 2 shows the observed spectral shapes for a constant covering factor $f_{\mathrm{c}}=90 \%$ and for varying optical depths of the covering medium (up to $128 N_{\mathrm{H}}^{(0)}$ ). As $N_{\mathrm{H}}$ is increased, the partial coverer removes most of the flux at soft energies and only the direct component remains visible.

Because absorption events are typically of rather short duration, the detailed spectral shape is often not directly observable. It is, however, possible to characterize the spectral 

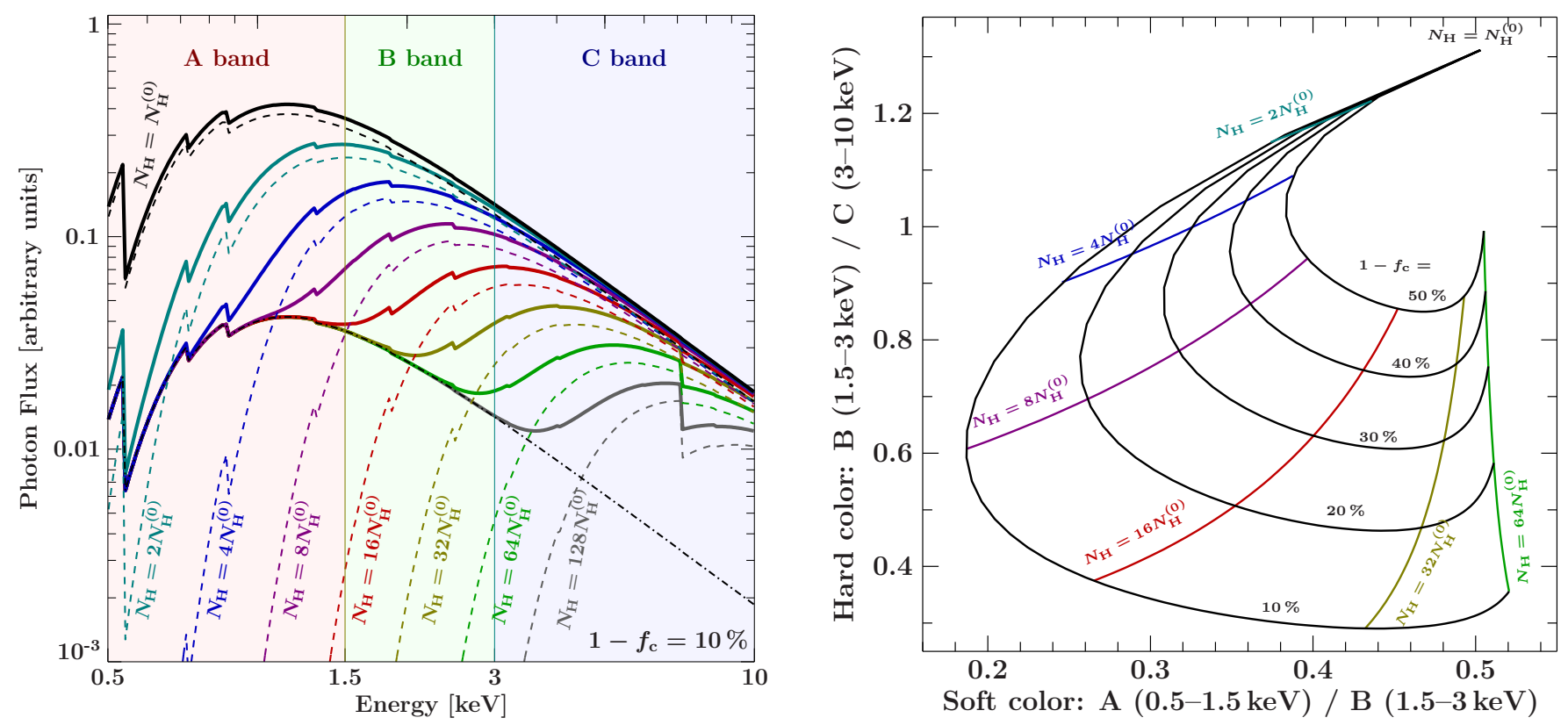

Fig. 2. Left panel: effect of partial absorption with increasing $N_{\mathrm{H}}$ at constant covering fraction with $N_{\mathrm{H}}^{(0)}=5.4 \times 10^{21} \mathrm{~cm}^{-2}$ after Paper I. Right panel: color-color diagram showing the tracks for different covering fractions and column densities of the second absorber. See text for details.

shape using X-ray colors or hardness ratios (see, e.g., Hanke et al. 2008; Nowak et al. 2011, for similar approaches). In this work, we define the X-ray hardness ratio as the ratio of the count rates in two energy bands. In order to be consistent with Paper I we define the ratio such that its value increases as the spectrum softens, so technically this ratio is a "softness ratio". We calculate hardness ratios using the fluxes measured in three energy bands - denoted as $A(0.5-1.5 \mathrm{keV} \equiv 24.8-8.27 \AA)$, $B(1.5-3.0 \mathrm{keV} \equiv 8.27-4.13 \AA)$, and $C \quad(3.0-10.0 \mathrm{keV} \equiv 4.13-$ $1.24 \AA$ ) with the ranges consistent with Paper II and the previous work of Nowak et al. (2011) - to characterize the spectral shape in this way.

The right-hand panel of Fig. 2 shows the locus of such colors for the spectral shape discussed above and several different covering fractions. For a constant $f_{\mathrm{c}}$, a characteristic track in the color-color diagram becomes apparent: At low $N_{\mathrm{H}} \sim N_{\mathrm{H}}^{(0)}$, the covered component dominates the spectrum and the source is found in the top right of the diagram. Since photoabsorption first influences the soft bands, increasing $N_{\mathrm{H}}$ decreases both, $A / B$ and $B / C$, and the source moves toward the bottom left of the diagram. At intermediate $N_{\mathrm{H}}$, the $A$ band is dominated by the (constant) uncovered fraction, but increasing $N_{\mathrm{H}}$ still decreases $B$ and $C$. For this reason, $A / B$ starts to increase again, while $B / C$ continues to decrease, such that the track of the source in the color-color-diagram starts to turn toward the right. When the contribution of the covered component to both $A$ and $B$ bands is almost negligible, $A / B$ remains constant, while $B / C$ increases with increasing $N_{\mathrm{H}}$. This behavior leads to a horizontal track in which the source moves to the right in the color-color diagram. Finally, for the largest $N_{\mathrm{H}}$ the entire covered fraction is removed from the observable spectrum such that we expect the $\mathrm{X}$-ray color to move asymptotically back to its unabsorbed value.

\subsection{Dip selection}

We now apply the ideas discussed above to Cyg X-1. Figure 3 shows the hardness ratios measured during the three observations considered in this work. The data show a behavior similar to that expected from partial covering: data outside of dips (orange data points) are barely absorbed; as the source enters deeper dips (bluer colors), it follows a track that resembles the theoretical tracks shown in Fig. 2, including a turning point where the soft color becomes softer, while the hard color barely changes.

To study the spectral shapes during different phases of the dips in more detail, we extract data from time intervals that correspond to various stages of dipping, i.e., phases of similar spectral shape. A direct comparison of the theoretical tracks derived from the simple partial covering model, however, shows deviations between the data and the tracks. These deviations could be indicative of changes in both the covering fraction and $N_{\mathrm{H}}$, ionization effects and because the underlying X-ray continuum is more complex than a simple absorbed power law. A detailed treatment of these effects, however, would require us to fully understand the ionization structure of the absorber, which is beyond the scope of this paper. We therefore determine the shape of the track empirically by fitting an empirical curve to the scatter plot. This curve is described through a parameterized polynomial of second degree for each of the two colors in the diagram. To find the polynomial coefficients, a $\chi^{2}$ minimization algorithm is used to optimize the shortest distance of each data point to the curve.

To select data from the dips, we first remove the non-dip data as defined in Paper $\mathrm{II}^{2}$. This ensures consistency with the earlier results from Paper II. For the remaining data, corresponding to various degrees of dipping, the goal is to find the highest possible resolution in terms of number of dipping stages, while maintaining a good enough signal-to-noise ratio $(\mathrm{S} / \mathrm{N})$ to be able to constrain the spectral fits well. To find this balance, we start out with a large number of 12 segments, which are chosen such that each slice contains roughly the same number of counts. Then, starting at the deepest dipping stage (bottom right-hand corner of the color-color diagram) we successively combine these small segments until the signal-to-noise ratio of the resulting spectra is

\footnotetext{
2 The simpler non-dip selection in Paper II was done using only the ratio $A / C$.
} 
(a)

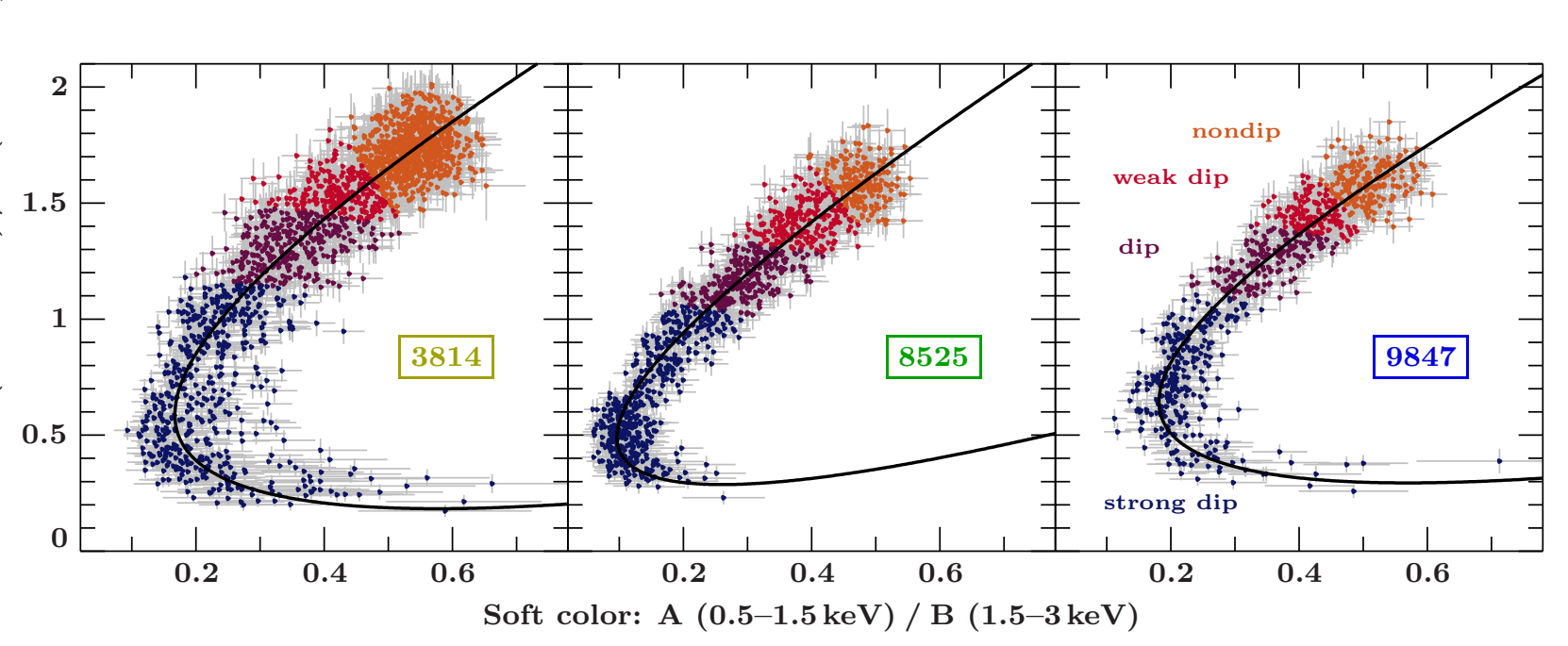

Fig. 3. Color-color diagrams for all three observations, showing hardness ratios calculated for soft, intermediate, and hard X-ray bands. As discussed in the text, the observed tracks are determined by absorption: following the track from the top right corner, representing the persistent flux, to the bottom left corner, showing dips, absorption increases and therefore both colors become harder. During the deepest dips, the softer color becomes softer again, while the hard color does not change. This behavior can be explained by partial covering (Sect. 2.2). The spectrum hardens considerably during these dips. The colors denote the four different dip stages (see right panel). The black line indicates our polynomial fit to the track; see Sect. 2.3 for details.

Table 2. Exposure time, $t_{\mathrm{exp}}$, and dip stage averaged count rates for the total observations and silicon $(1.605-2.045 \mathrm{keV} \equiv 7.725-6.063 \AA)$ and sulfur $(2.295-2.7 \mathrm{keV} \equiv 5.402-4.6 \AA)$ bands for all observations.

\begin{tabular}{|c|c|c|c|c|c|}
\hline & & \multicolumn{4}{|c|}{ Counts } \\
\hline & & $\begin{array}{l}\quad t_{\exp } \\
(\mathrm{ks})\end{array}$ & $\begin{array}{l}\text { Total } \\
\left(10^{3} \mathrm{c}\right.\end{array}$ & $\begin{array}{c}\mathrm{Si} \\
\text { unts }\end{array}$ & ${ }_{1}{ }^{S}$ \\
\hline \multirow{4}{*}{3814} & Non-dip & 21.096 & 1838 & 37 & 92 \\
\hline & Weak dip & 6.095 & 445 & 10 & 26 \\
\hline & Dip & 7.345 & 461 & 13 & 31 \\
\hline & Strong dip & 12.559 & 458 & 21 & 49 \\
\hline \multirow{4}{*}{8525} & Non-dip & 5.079 & 450 & 11 & 22 \\
\hline & Weak dip & 5.313 & 394 & 11 & 26 \\
\hline & Dip & 6.834 & 406 & 13 & 28 \\
\hline & Strong dip & 12.200 & 401 & 20 & 41 \\
\hline \multirow{4}{*}{9847} & Non-dip & 4.880 & 499 & 12 & 24 \\
\hline & Weak dip & 3.273 & 294 & 8 & 16 \\
\hline & Dip & 4.083 & 307 & 9 & 19 \\
\hline & Strong dip & 6.618 & 303 & 15 & 29 \\
\hline
\end{tabular}

sufficient to detect all possible lines of the Si and S series, but keep the number of segments for this as low as possible to be able to distinguish a greater number of dip stages. These combined segments constitute the selection for the deepest dipping stage for further analysis. Subsequently, the selection of the next dipping stages follow the same approach until all 12 segments are sorted.

For all observations we obtain the best results by defining three dipping stages in addition to the non-dip phase, each consisting of four of the smaller segments. Consequently, the three stages each have roughly the same number of counts within an observation. The four dipping stages for each observation are classified as "non-dip", "weak dip", "dip", and "strong dip" (see Fig. 3 and Table 2 for the count rates and exposure of each dip stage).

\section{Spectral evolution from non-dip to dip}

Figure 4 shows the evolution of the spectrum during dipping for ObsID 8525. In addition to the general change in spectral shape due to photoelectric absorption, we see strong changes between the individual dipping stages in the region between 1.6 and $2.7 \mathrm{keV}(7.7-4.6 \AA)$, where absorption lines of silicon and sulfur ions are the most prominent spectral features (box in Fig. 4, see also Miškovičová et al. 2011). We therefore concentrate on these $\mathrm{Si}$ and $\mathrm{S}$ lines.

\subsection{Si and S regions}

For a detailed analysis of the $\mathrm{Si}$ and $\mathrm{S}$ absorption lines, we take into account the \pm 1 order HEG and MEG spectra. We combine both spectra for the HEG and the MEG, and fit the spectra without rebinning them. For clarity reasons, all figures show the combined HEG and MEG spectra, where the higher resolution of the HEG is rebinned to the lower resolution of the MEG. This combination of HEG and MEG, however, only applies to the display of the data, not to the actual fitting.

As the emphasis of this paper is on the behavior of the $\mathrm{Si}$ and $\mathrm{S}$ lines, we do not attempt to model the broadband Chandra continuum or the continuum absorption, but rather describe the local spectra in the $\mathrm{Si}(1.605-2.045 \mathrm{keV} \equiv 7.725-6.063 \AA)$ and the $\mathrm{S}(2.295-2.7 \mathrm{keV} \equiv 5.402-4.6 \AA)$ regions. These regions are narrow enough in energy that the curvature due to the absorption is negligible. We can therefore describe the local absorbed continuum by a simple power law; obviously, the photon indices of the continuum are different in both bands.

A further complication is that as a consequence of the source brightness the non-dip, weak dip, and to a lesser extent also the dip spectra are affected by slight pileup. The major effect that pileup has on our narrowband data is a change in count rate, which is seen as a change in the relative flux normalization of both spectra, as pileup affects the MEG more strongly than the HEG because of the significantly lower spectral 


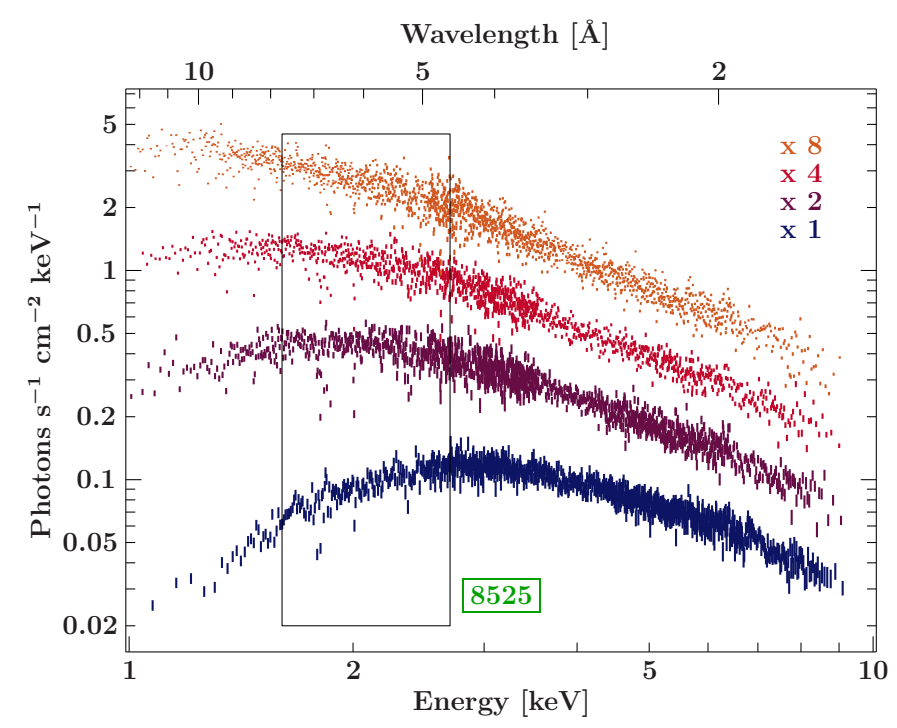

Fig. 4. Rebinned HEG spectra of the different dipping stages of ObsID 8525. For clarity, the non-dip spectrum (orange, $S / N \geq 13$ ) is scaled by a factor of 8 , the weak dip spectrum (red, $S / N \geq 15$ ) by a factor of 4 , and the dip spectrum (violet, $S / N \geq 15$ ) by a factor of 2 . The strong dip spectrum (blue, $S / N \geq 17$ ) is not scaled. The box denotes the region containing the $\mathrm{Si}$ and $\mathrm{S}$ lines. The color scheme is the same as in Figs. 1 and 3 .

resolution ${ }^{3}$. We can compensate for this effect by including a multiplicative, detector dependent constant in the spectral modeling. Because of the narrow energy bands considered here, a further pileup correction is not necessary. The pileup fraction is a slowly changing function of energy such that it does not appreciably affect the equivalent width of the lines considered in this work; because of the nature of X-ray gratings, line energies are not affected. See Hanke et al. (2009) for a discussion of pileup effects in the non-dip spectrum of Cyg X-1.

The local continuum being accounted for (see Table 3 for parameters), we model the absorption lines in both regions using additive Gaussian line profiles (Fig. 5). These profiles give a slightly better description of the line shapes than Voigt profiles. For the absorption lines, the line width $\sigma$ was frozen at $1.2 \mathrm{eV}$ (silicon) and $1.8 \mathrm{eV}$ (sulfur), well below the detector resolution. Lines were identified using energies from Hell et al. (2016), Porquet et al. (2010), and from AtomDB, version 2.0.04 . We find blended line complexes for L-shell ions, He w lines for He-like ions, and Ly $\alpha$ lines for $\mathrm{H}$-like ions of both silicon and sulfur. In addition to the $\mathrm{K} \alpha$ absorption line series, there is the forbidden He-like Si XIII z line in emission $(1.8394 \mathrm{keV} \equiv 6.7405 \AA$, $1 \mathrm{~s} 2 \mathrm{~s}{ }^{3} \mathrm{~S}_{1} \rightarrow 1 \mathrm{~s}^{2}{ }^{1} \mathrm{~S}_{0}$; Porquet et al. 2010); this line is sometimes also called the He-like Si XIII f line. We note that there is a degeneracy between the Be-like Si XI and Li-like Si XII absorption lines at $1.8275 \mathrm{keV} \equiv 6.7844 \AA$ and $1.8450 \mathrm{keV} \equiv 6.7200 \AA$ (both energies from Hell et al. 2016) and the forbidden He-like Si XIII z emission line. This increases the uncertainty of the respective fit values. Finally, in the strong dip spectrum of ObsID 9847, there are indications for a O-like S IX K $\alpha$ line, which appears once the HEG data are rebinned to match the (unbinned) MEG grid and both are combined for display. This line is too weak for us to be able to constrain its parameters such

\footnotetext{
3 See "The Chandra ABC Guide to Pileup", CXC, 2010, http: //cxc. harvard.edu/ciao/download/doc/pileup_abc.pdf

4 See http://www.atomdb.org; line energies without a reference refer to AtomDB.
}

that we only claim a tentative detection of O-like S IX. The values for central line energies and equivalent widths of all detected silicon and sulfur absorption lines can be found in Tables 4 and 5 . We note that the lower ionization stages (N-like and O-like ions) only appear deeper in the dips. This hints toward a shell-like ionization structure of the clumps with a hot, highly ionized surface and cooler and less ionized parts in the core.

In addition to these lines from $\mathrm{S}$ and $\mathrm{Si}$ we also find an Al XIII Ly $\alpha^{5}$ absorption line $(1.7285 \mathrm{keV} \equiv 7.1729 \AA), \mathrm{Mg} \mathrm{He} \gamma$ $\left(1.6591 \mathrm{keV} \equiv 7.4730 \AA, 1 \mathrm{~s}^{2}{ }^{1} \mathrm{~S}_{0}-1 \mathrm{~s} 4 \mathrm{p}^{1} \mathrm{P}_{1}\right)$ and $\mathrm{He} \delta(1.6961 \mathrm{keV}$ $\left.\equiv 7.3100 \AA, 1 \mathrm{~s}^{2}{ }^{1} \mathrm{~S}_{0}-1 \mathrm{~s} 5 \mathrm{p}^{1} \mathrm{P}_{1}\right)$ absorption lines, and we detect $\operatorname{Mg}$ XII Ly $\beta(1.7447 \mathrm{keV} \equiv 7.1063 \AA)$ and, in some cases, a hint of $\operatorname{Mg} \operatorname{Ly} \delta(1.8843 \mathrm{keV} \equiv 6.5799 \AA)$ blended with an Fe XXIV transition $\left(1.8851 \mathrm{keV} \equiv 5.5771 \AA, 1 \mathrm{~s}^{2} 2 \mathrm{~s}^{2} \mathrm{~S}_{1 / 2}-1 \mathrm{~s}^{2} 7 \mathrm{p}^{2} \mathrm{P}_{3 / 2}\right)$. These lines imply the presence of $\mathrm{MgLy} \gamma$ at $1.840 \mathrm{keV} \equiv 6.738 \AA$, i.e., blended with He-like $\mathrm{Siz}$. We expect $\mathrm{MgLy} \gamma$ to be very weak but it may slightly contaminate our measurements of the $\mathrm{SiHe}$ z and the Li-like Si XII line, thus further contributing to the uncertainty of the fit values.

\subsubsection{Equivalent widths or line strengths and column densities}

The equivalent widths of the silicon and sulfur lines vary around $1 \mathrm{eV}$ and have rather large error bars for the weaker lines (Table 5). We note that the He-like Si XIII z emission line additionally increases the uncertainties of the Si XI and Si XII lines. Comparing the lines in the different dipping stages reveals that the line strengths of the low charge states - and thus the respective column densities - increase with dipping, while those of the higher charge states decrease (Fig. 6). This shift in charge balance alone is already strong evidence supporting the theory of a highly structured medium where clumps of colder, denser material are embedded in a highly ionized plasma (Oskinova et al. 2012; Sundqvist \& Owocki 2013, and references therein).

For optically thin absorption lines, the equivalent width directly translates into the column density of the parent ion, $N_{i}$. In this section and in the following we use the convention that the equivalent width of absorption lines is negative and that of emission lines is positive. We derive the column densities for all $\mathrm{Si}$ and $\mathrm{S}$ ions visible in our spectra from (Paper I, Eq. (11))

$N_{i}=\frac{m_{\mathrm{e}} c^{2}\left|W_{\lambda}\right|}{\pi e^{2} f_{i j} \lambda_{0}^{2}}=\frac{9.11 \times 10^{15} \mathrm{~cm}^{-2}}{f_{i j}} \frac{\left|W_{\mathrm{E}}\right|}{\mathrm{eV}}$,

where $W_{\lambda}$ and $W_{\mathrm{E}}$ are the equivalent width in wavelength and in energy space, and where $f_{i j}$ is the oscillator strength, taken as the sum of all transitions blending into the absorption line of the respective ion. We use the compilation of Verner et al. (1996) for H-like ions, and the compilation of Palmeri et al. (2008) for all other ionization stages. These oscillator strengths include Auger damping, which is important at the densities expected. All other symbols have their usual meanings.

Summing the column densities for all ions of an element then yields a lower limit for the total column density of the element. For comparison with the continuum fitting values, the abundance of the element can then be utilized to convert this column density

5 Lines denoted as part of the Lyman series typically have two unresolved components with the configuration of $1 \mathrm{~s}_{1 / 2}{ }^{2} \mathrm{~S}_{1 / 2}-\mathrm{np}_{3 / 2}{ }^{2} \mathrm{P}_{3 / 2}$ and $1 \mathrm{~s}_{1 / 2}{ }^{2} \mathrm{~S}_{1 / 2}-\mathrm{np}_{1 / 2}{ }^{2} \mathrm{P}_{1 / 2}$, respectively. The labels $\alpha, \beta, \gamma$ etc. represent the principal quantum number $n=2,3,4, \ldots$ The line energy given in the text corresponds to the mean energy of the components, weighted with the respective statistical weight (2:1). 
Table 3. Detector constants and continuum parameters for all observations.

\begin{tabular}{|c|c|c|c|c|}
\hline 3814 & & Detector constant & $\begin{array}{l}\text { Power law norm } \\
\text { (photons } \mathrm{keV}^{-1} \mathrm{~cm}^{-2} \mathrm{~s}^{-1} \text { ) }\end{array}$ & Photon index \\
\hline \multirow[t]{4}{*}{ Si region } & Non-dip & $0.779 \pm 0.005$ & $0.709_{-0.016}^{+0.017}$ & $1.12 \pm 0.04$ \\
\hline & Weak dip & $0.830 \pm 0.010$ & $0.493_{-0.023}^{-0.010}$ & $0.84 \pm 0.08$ \\
\hline & Dip & $0.873 \pm 0.010$ & $0.306_{-0.014}^{+0.015}$ & $0.43 \pm 0.08$ \\
\hline & Strong dip & $0.958_{-0.013}^{+0.014}$ & $0.057 \pm 0.004$ & $-0.83 \pm 0.10$ \\
\hline \multirow[t]{4}{*}{$S$ region } & Non-dip & $0.948 \pm 0.010$ & $1.14_{-0.12}^{+0.13}$ & $1.60 \pm 0.12$ \\
\hline & Weak dip & $0.966 \pm 0.018$ & $0.87_{-0.18}^{-0.12}$ & $1.42 \pm 0.25$ \\
\hline & Dip & $0.985_{-0.017}^{+0.018}$ & $0.74_{-0.15}^{+0.18}$ & $1.37 \pm 0.23$ \\
\hline & Strong dip & $1.010 \pm 0.018$ & $0.088_{-0.018}^{+0.023}$ & $-0.31 \pm 0.25$ \\
\hline 8525 & & Detector constant & $\begin{array}{l}\text { Power law norm } \\
\left(\text { photons } \mathrm{keV}^{-1} \mathrm{~cm}^{-2} \mathrm{~s}^{-1} \text { ) }\right.\end{array}$ & $\begin{array}{l}\text { Photon index } \\
\left(\times 10^{-4}\right)\end{array}$ \\
\hline \multirow[t]{4}{*}{ Si region } & Non-dip & $0.791 \pm 0.009$ & $0.63 \pm 0.04$ & $0.95 \pm 0.09$ \\
\hline & Weak dip & $0.832_{-0.010}^{+0.011}$ & $0.457_{-0.023}^{+0.025}$ & $0.70 \pm 0.09$ \\
\hline & Dip & $0.900 \pm 0.012$ & $0.247_{-0.013}^{+0.014}$ & $0.20 \pm 0.09$ \\
\hline & Strong dip & $0.989 \pm 0.016$ & $0.0368_{-0.0023}^{+0.0025}$ & $-1.27 \pm 0.11$ \\
\hline \multirow[t]{4}{*}{ S region } & Non-dip & $1.000_{-0.021}^{+0.022}$ & $1.04_{-0.20}^{+0.25}$ & $1.45 \pm 0.23$ \\
\hline & Weak dip & $0.960 \pm 0.020$ & $0.56_{-0.13}^{+0.17}$ & $0.87 \pm 0.28$ \\
\hline & Dip & $0.987 \pm 0.019$ & $0.44_{-0.10}^{+0.12}$ & $0.84 \pm 0.26$ \\
\hline & Strong dip & $1.022_{-0.019}^{+0.020}$ & $0.082_{-0.017}^{+0.025}$ & $-0.33_{-0.26}^{+0.29}$ \\
\hline 9847 & & Detector constant & $\begin{array}{l}\text { Power law norm } \\
\text { (photons } \mathrm{keV}^{-1} \mathrm{~cm}^{-2} \mathrm{~s}^{-1} \text { ) }\end{array}$ & $\begin{array}{l}\text { Photon index } \\
\left(\times 10^{-4}\right)\end{array}$ \\
\hline \multirow[t]{4}{*}{ Si region } & Non-dip & $0.728 \pm 0.008$ & $0.84 \pm 0.04$ & $1.08 \pm 0.08$ \\
\hline & Weak dip & $0.773 \pm 0.012$ & $0.62 \pm 0.04$ & $0.84 \pm 0.11$ \\
\hline & Dip & $0.846 \pm 0.013$ & $0.374_{-0.024}^{+0.026}$ & $0.43_{-0.11}^{+0.12}$ \\
\hline & Strong dip & $0.970 \pm 0.018$ & $0.062 \pm 0.005$ & $-1.12 \pm 0.12$ \\
\hline \multirow[t]{4}{*}{ S region } & Non-dip & $0.925 \pm 0.019$ & $1.29_{-0.27}^{+0.26}$ & $1.50_{-0.25}^{+0.20}$ \\
\hline & Weak dip & $0.958_{-0.029}^{+0.030}$ & $1.4_{-0.4}^{+0.5}$ & $1.74_{-0.30}^{+0.29}$ \\
\hline & Dip & $0.978_{-0.028}^{+0.029}$ & $0.64_{-0.16}^{+0.20}$ & $1.01_{-0.30}^{+0.29}$ \\
\hline & Strong dip & $1.003_{-0.029}^{+0.030}$ & $0.16_{-0.04}^{+0.06}$ & $0.1 \pm 0.4$ \\
\hline
\end{tabular}

Notes. The power law fits are applied to a very local continuum $(1.605-2.045 \mathrm{keV} \equiv 7.725-6.063 \AA$ for the $\mathrm{Si}$ region and $2.295-2.7 \mathrm{keV} \equiv 5.402-$ 4.6 $\AA$ for the $\mathrm{S}$ region). The detector constant accounts for the different flux normalizations of the HEG and the MEG.

into a corresponding $N_{\mathrm{H}}$ value. The values listed in Tables 6 and 7 list two $N_{\mathrm{H}}$ values. One value is based on the solar abundances as summarized by Wilms et al. (2000). In our second conversion we consider that in their analysis of the optical spectrum of HDE 226868 Herrero et al. (1995) found that helium is overabundant by a factor of 2.53 with respect to the solar value. Assuming that metals scale with the same factor as helium with respect to hydrogen, we scale the solar abundances of Wilms et al. (2000) by this factor and use these corrected abundances to derive the corresponding hydrogen column.

Figure 6 shows how the single ion column densities vary with depth of the dip. As we enter the dip, for the lowest ionization states (Fig. 6, bottom panels) the columns increase toward the deepest dip stages. This trend reverses for the highest ionization states (Fig. 6, top panels). This behavior is also indicative of an absorber with a layered ionization structure, i.e., a medium whose outer regions are more highly ionized than the central core region.
For the non-dip data, column densities of H-like and He-like silicon have already been presented in Paper I. Despite the fact that our dip selection criterion for the non-dip data has not changed, there is a slight difference in the equivalent width determined for He-like Si XIII, where Paper I finds an equivalent width of $-1.73 \mathrm{eV}$, while we find $-1.92 \mathrm{eV}$, resulting in a $\sim 10 \%$ difference in the column. Both values are still in agreement within their uncertainties. The difference in equivalent width for $\mathrm{H}$-like Si XIV, on the other hand, is a surprising factor 1.3. A possible explanation is that the non-dip data allowed a global fit, including interstellar absorption, more than a hundred lines, and a pileup correction. The three dip stages, however, do not have enough signal to constrain such a model, and consequently for consistency we also model the non-dip data with local fits. Differences at the $15 \%$ level would therefore be expected owing to these different analysis approaches. Taking into account these additional $15 \%$, the H-like Si XIV columns are also consistent within their uncertainties. 

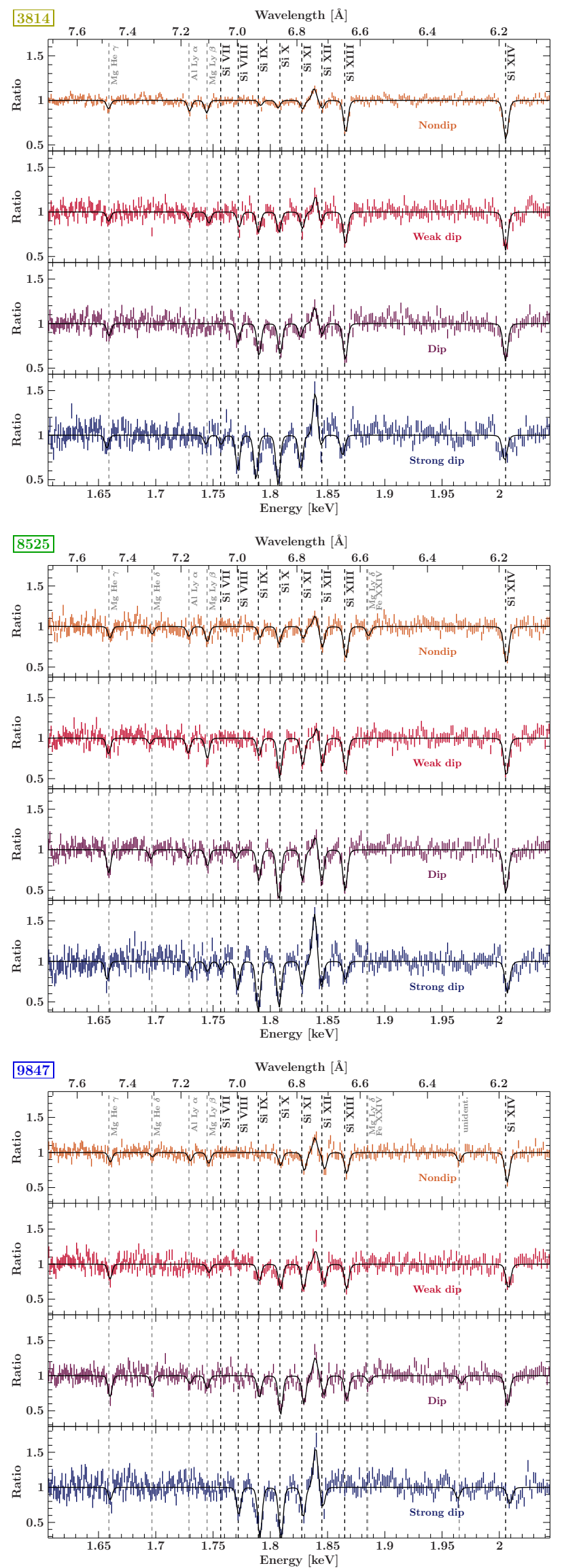
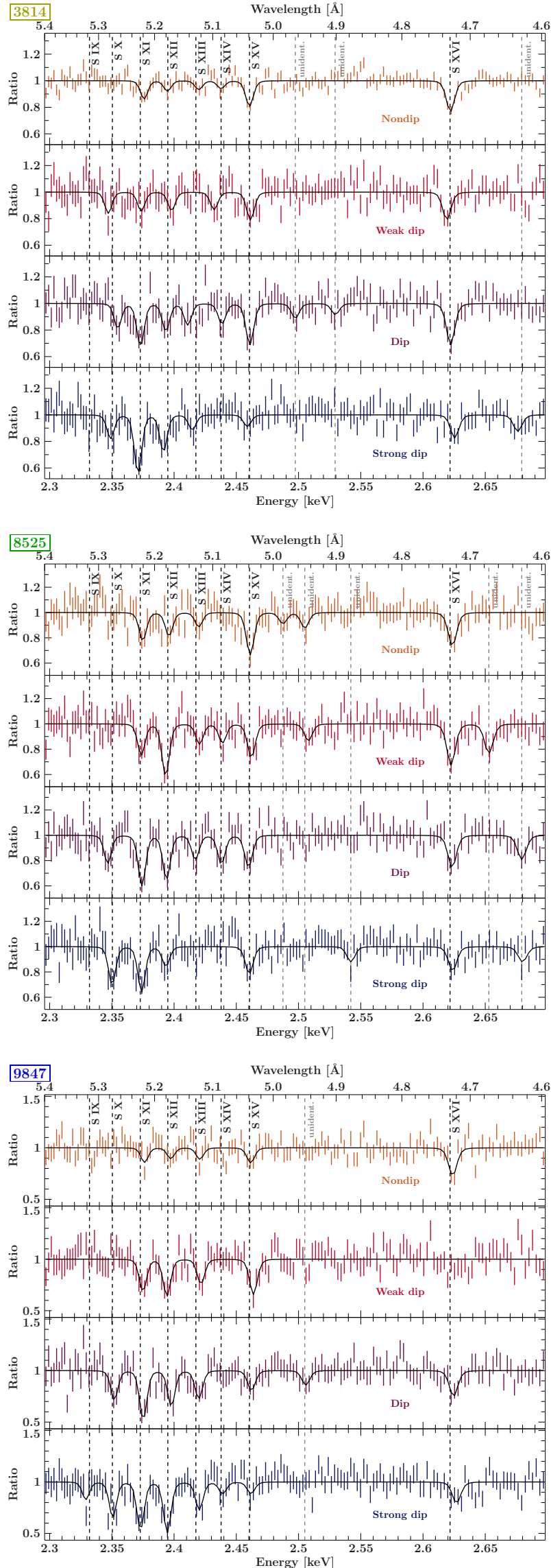

Fig. 5. Evolution of silicon (left column) and sulfur (right column) lines with dipping stages for the three observations considered (from top to bottom). The panels show combined HEG and MEG \pm 1 st spectra relative to their respective continua. The solid black line shows the line fits. 
M. Hirsch et al.: Chandra X-ray spectroscopy of the focused wind in the Cygnus X-1 system. III.

Table 4. Observed silicon and sulfur line centers for all observations.

\begin{tabular}{|c|c|c|c|c|c|c|}
\hline \multirow{2}{*}{3814} & \multirow{2}{*}{\multicolumn{2}{|c|}{ Ion }} & \multicolumn{4}{|c|}{$E_{\text {obs }}(\mathrm{keV})$} \\
\hline & & & Non-dip & Weak dip & Dip & Strong dip \\
\hline & H-like & Si XIV & $2.00574(23)$ & $2.0055(5)$ & $2.0054(6)$ & $2.0048(26)$ \\
\hline & He-like & Si XIII & $1.86588(22)$ & $1.8656(6)$ & $1.8655(4)$ & $1.8632(11)$ \\
\hline & Li-like & Si XII & $1.8452(17)$ & $1.8440(28)$ & $1.8451(26)$ & $1.8437(14)$ \\
\hline & Be-like & Si XI & $1.8283(8)$ & $1.8280(7)$ & $1.8264(19)$ & $1.8267(7)$ \\
\hline & B-like & Si X & $1.8069(17)$ & $1.8077(11)$ & $1.8086(8)$ & $1.80678(43)$ \\
\hline & C-like & Si IX & $1.7916(13)$ & $1.7897(8)$ & $1.7902(9)$ & $1.7875(6)$ \\
\hline & N-like & Si VIII & - & $1.7728(8)$ & $1.7719(7)$ & $1.7717(5)$ \\
\hline & O-like & Si VII & - & - & - & $1.7571(15)$ \\
\hline & H-like & S XVI & $2.6220(9)$ & 2.6191(22) & $2.6222(11)$ & $2.6254(20)$ \\
\hline & He-like & S XV & $2.4606(9)$ & $2.4614(24)$ & $2.4608(12)$ & $2.459(6)$ \\
\hline & Li-like & S XIV & $2.4378(22)$ & $2.4321(30)$ & $2.4385(19)$ & - \\
\hline & Be-like & S XIII & $2.420(4)$ & - & $2.4110(88)$ & $2.4147(29)$ \\
\hline & B-like & S XII & $2.3947(25)$ & $2.3980(24)$ & $2.3934(21)$ & $2.3914(19)$ \\
\hline & C-like & S XI & $2.3759(16)$ & $2.374(7)$ & $2.3730(18)$ & $2.3710(11)$ \\
\hline & N-like & $\mathrm{SX}$ & - & $2.3472(23)$ & $2.3548(22)$ & $2.3490(35)$ \\
\hline & O-like & S IX & - & - & - & - \\
\hline \multirow{18}{*}{8525} & \multirow{2}{*}{\multicolumn{2}{|c|}{ Ion }} & \multicolumn{4}{|c|}{$E_{\text {obs }}(\mathrm{keV})$} \\
\hline & & & Non-dip & Weak dip & Dip & Strong dip \\
\hline & H-like & Si XIV & $2.0061(5)$ & $2.0061(5)$ & $2.0057(4)$ & $2.0070(7)$ \\
\hline & He-like & Si XIII & $1.8660(5)$ & $1.8659(5)$ & $1.8653(4)$ & $1.8656(11)$ \\
\hline & Li-like & Si XII & $1.8454(8)$ & $1.8452(18)$ & $1.8448(6)$ & $1.8446(9)$ \\
\hline & Be-like & Si XI & $1.8289(10)$ & $1.8284(5)$ & $1.8281(4)$ & $1.8278(6)$ \\
\hline & B-like & Si X & $1.8078(9)$ & $1.80823(47)$ & $1.80748(31)$ & $1.80772(41)$ \\
\hline & C-like & Si IX & $1.7911(13)$ & $1.7903(9)$ & $1.7901(7)$ & $1.78944(38)$ \\
\hline & N-like & Si VIII & - & - & $1.7705(27)$ & $1.7716(6)$ \\
\hline & O-like & Si VII & - & - & - & $1.7568(21)$ \\
\hline & H-like & S XVI & $2.6236(17)$ & $2.6225(13)$ & $2.6234(18)$ & $2.6238(22)$ \\
\hline & He-like & S XV & $2.4612(11)$ & $2.4621(17)$ & $2.4598(15)$ & $2.4604(19)$ \\
\hline & Li-like & S XIV & - & $2.4389(24)$ & $2.4380(17)$ & - \\
\hline & Be-like & S XIII & $2.4198(27)$ & $2.4204(23)$ & $2.4171(16)$ & - \\
\hline & B-like & S XII & $2.3959(29)$ & $2.3932(9)$ & $2.3940(11)$ & $2.3934(23)$ \\
\hline & C-like & S XI & $2.3749(17)$ & $2.3737(18)$ & $2.3742(10)$ & $2.3739(20)$ \\
\hline & N-like & $\mathrm{SX}$ & - & - & $2.3467(17)$ & $2.3502(15)$ \\
\hline & O-like & S IX & - & - & - & - \\
\hline \multirow{18}{*}{9847} & \multirow{2}{*}{\multicolumn{2}{|c|}{ Ion }} & \multicolumn{4}{|c|}{$E_{\text {obs }}(\mathrm{keV})$} \\
\hline & & & Non-dip & Weak dip & Dip & Strong dip \\
\hline & H-like & Si XIV & $2.0069(4)$ & $2.0079(10)$ & $2.0070(7)$ & $2.0092(12)$ \\
\hline & He-like & Si XIII & $1.8668(6)$ & $1.8667(6)$ & $1.8668(7)$ & - \\
\hline & Li-like & Si XII & $1.8474(6)$ & $1.8468(10)$ & $1.8468(9)$ & $1.8459(10)$ \\
\hline & Be-like & Si XI & $1.8297(6)$ & $1.8291(5)$ & $1.8293(6)$ & $1.8287(5)$ \\
\hline & B-like & Si X & $1.8089(9)$ & $1.8090(8)$ & $1.8090(5)$ & $1.80928(37)$ \\
\hline & C-like & Si IX & - & $1.7904(13)$ & $1.7905(14)$ & $1.79092(37)$ \\
\hline & N-like & Si VIII & - & - & - & $1.7725(5)$ \\
\hline & O-like & Si VII & - & - & - & - \\
\hline & H-like & S XVI & $2.6238(12)$ & - & $2.6246(19)$ & $2.6269(39)$ \\
\hline & He-like & S XV & $2.4615(23)$ & $2.4637(14)$ & $2.4621(30)$ & $2.4618(36)$ \\
\hline & Li-like & S XIV & - & - & - & $2.4391(33)$ \\
\hline & Be-like & S XIII & $2.4207(23)$ & $2.4216(16)$ & $2.4198(25)$ & $2.4202(17)$ \\
\hline & B-like & S XII & $2.397(4)$ & $2.3942(16)$ & $2.3978(15)$ & $2.3945(11)$ \\
\hline & C-like & S XI & $2.3763(20)$ & $2.3750(18)$ & $2.3752(11)$ & $2.3730(14)$ \\
\hline & N-like & $\mathrm{Sx}$ & - & - & $2.3520(17)$ & $2.3508(22)$ \\
\hline & O-like & S IX & - & - & - & $2.3291(26)$ \\
\hline
\end{tabular}


Table 5. Evolution of the equivalent widths of the silicon and sulfur lines during the dipping stages for all observations.

\begin{tabular}{|c|c|c|c|c|c|c|}
\hline \multirow{2}{*}{3814} & \multirow{2}{*}{\multicolumn{2}{|c|}{ Ion }} & \multicolumn{4}{|c|}{ Equivalent width (eV) } \\
\hline & & & Non-dip & Weak dip & Dip & Strong dip \\
\hline & H-like & Si XIV & $-2.48 \pm 0.14$ & $-2.42_{-0.28}^{+0.29}$ & $-2.38_{-0.28}^{+0.29}$ & $-1.7 \pm 0.4$ \\
\hline & He-like & Si XIII & $-1.92 \pm 0.14$ & $-1.93 \pm 0.28$ & $-2.16 \pm 0.27$ & $-1.2 \pm 0.4$ \\
\hline & Li-like & Si XII & $-0.46 \pm 0.16$ & $-0.7_{-1.0}^{+0.4}$ & $-0.6 \pm 0.4$ & $-0.8_{-0.6}^{+0.5}$ \\
\hline & Be-like & Si XI & $-0.49 \pm 0.13$ & $-0.95 \pm 0.25$ & $-0.77_{-0.26}^{+0.27}$ & $-1.88 \pm 0.28$ \\
\hline & B-like & Si $X$ & $-0.42 \pm 0.14$ & $-1.13_{-0.25}^{+0.26}$ & $-1.76 \pm 0.24$ & $-2.81 \pm 0.26$ \\
\hline & C-like & Si IX & $-0.28 \pm 0.14$ & $-1.13 \pm 0.25$ & $-1.80_{-0.24}^{+0.25}$ & $-2.48_{-0.26}^{+0.27}$ \\
\hline & N-like & Si VIII & - & $-0.83 \pm 0.26$ & $-1.05 \pm 0.26$ & $-1.94 \pm 0.29$ \\
\hline & O-like & Si VII & - & - & - & $-0.5 \pm 0.4$ \\
\hline & H-like & SXVI & $-2.2 \pm 0.4$ & $-1.9 \pm 0.8$ & $-2.9 \pm 0.7$ & $-1.6 \pm 0.7$ \\
\hline & He-like & S XV & $-1.6 \pm 0.4$ & $-1.8 \pm 0.7$ & $-2.7 \pm 0.6$ & $-0.8 \pm 0.7$ \\
\hline & Li-like & S XIV & $-0.5 \pm 0.4$ & $-1.1 \pm 0.7$ & $-1.3 \pm 0.7$ & - \\
\hline & Be-like & S XIII & $-0.6 \pm 0.4$ & - & $-1.3 \pm 0.7$ & $-1.0 \pm 0.7$ \\
\hline & B-like & S XII & $-0.6 \pm 0.4$ & $-1.1 \pm 0.7$ & $-1.7 \pm 0.7$ & $-2.3 \pm 0.7$ \\
\hline & C-like & S XI & $-1.1 \pm 0.4$ & $-1.2 \pm 0.9$ & $-2.6 \pm 0.7$ & $-3.6 \pm 0.7$ \\
\hline & N-like & $\mathrm{Sx}$ & - & $-1.3 \pm 0.8$ & $-1.5 \pm 0.8$ & $-1.4 \pm 0.8$ \\
\hline & O-like & S IX & - & - & - & - \\
\hline \multirow{18}{*}{8525} & \multirow{2}{*}{\multicolumn{2}{|c|}{ Ion }} & \multicolumn{4}{|c|}{ Equivalent width (eV) } \\
\hline & & & Non-dip & Weak dip & Dip & Strong dip \\
\hline & H-like & Si XIV & $-2.58_{-0.28}^{+0.29}$ & $-2.67 \pm 0.30$ & $-3.07 \pm 0.28$ & $-2.2 \pm 0.4$ \\
\hline & He-like & Si XIII & $-2.08 \pm 0.28$ & $-2.18 \pm 0.29$ & $-2.67 \pm 0.28$ & $-1.2 \pm 0.4$ \\
\hline & Li-like & Si XII & $-1.3 \pm 0.4$ & $-1.8 \pm 0.4$ & $-2.1 \pm 0.4$ & $-1.7 \pm 0.4$ \\
\hline & Be-like & Si XI & $-0.76 \pm 0.26$ & $-1.67 \pm 0.25$ & $-1.94 \pm 0.25$ & $-1.5 \pm 0.4$ \\
\hline & B-like & Si X & $-1.10 \pm 0.25$ & $-2.46_{-0.23}^{+0.24}$ & $-3.11_{-0.21}^{+0.22}$ & $-2.88 \pm 0.28$ \\
\hline & C-like & Si IX & $-0.66 \pm 0.25$ & $-1.10 \pm 0.26$ & $-1.94 \pm 0.25$ & $-3.11_{-0.26}^{+0.27}$ \\
\hline & N-like & Si VIII & - & - & $-0.52 \pm 0.30$ & $-1.8 \pm 0.4$ \\
\hline & O-like & Si VII & - & - & - & $-0.5 \pm 0.4$ \\
\hline & H-like & S XVI & $-2.5 \pm 0.8$ & $-3.1 \pm 0.8$ & $-2.4 \pm 0.8$ & $-1.8 \pm 0.8$ \\
\hline & He-like & S XV & $-2.9 \pm 0.7$ & $-2.3 \pm 0.7$ & $-2.2 \pm 0.7$ & $-1.9 \pm 0.7$ \\
\hline & Li-like & S XIV & - & $-1.3_{-0.7}^{+0.8}$ & $-1.9 \pm 0.7$ & - \\
\hline & Be-like & S XIII & $-0.9 \pm 0.7$ & $-1.3 \pm 0.8$ & $-1.6 \pm 0.7$ & - \\
\hline & B-like & S XII & $-1.5 \pm 0.7$ & $-3.4 \pm 0.7$ & $-2.9 \pm 0.7$ & $-1.5 \pm 0.8$ \\
\hline & C-like & S XI & $-1.8 \pm 0.7$ & $-2.0 \pm 0.8$ & $-3.2 \pm 0.7$ & $-2.9 \pm 0.7$ \\
\hline & N-like & $\mathrm{SX}$ & - & - & $-1.8_{-0.7}^{+0.8}$ & $-2.4_{-0.7}^{+0.8}$ \\
\hline & O-like & S IX & - & - & - & - \\
\hline \multirow{18}{*}{9847} & \multirow{2}{*}{\multicolumn{2}{|c|}{ Ion }} & \multicolumn{4}{|c|}{ Equivalent width (eV) } \\
\hline & & & Non-dip & Weak dip & Dip & Strong dip \\
\hline & H-like & Si XIV & $-2.34_{-0.27}^{+0.28}$ & $-1.9 \pm 0.4$ & $-2.4 \pm 0.4$ & $-1.4 \pm 0.5$ \\
\hline & He-like & Si XIII & $-1.56 \pm 0.28$ & $-1.9 \pm 0.4$ & $-1.8 \pm 0.4$ & - \\
\hline & Li-like & Si XII & $-1.3 \pm 0.4$ & $-1.6 \pm 0.5$ & $-1.5_{-0.5}^{+0.4}$ & $-1.5 \pm 0.5$ \\
\hline & Be-like & Si XI & $-1.31 \pm 0.24$ & $-1.82 \pm 0.29$ & $-1.91 \pm 0.29$ & $-2.2 \pm 0.4$ \\
\hline & B-like & Si $\mathrm{X}$ & $-0.92 \pm 0.24$ & $-1.76 \pm 0.29$ & $-2.78 \pm 0.26$ & $-3.86_{-0.26}^{+0.27}$ \\
\hline & C-like & Si IX & - & $-1.2 \pm 0.4$ & $-1.55_{-0.30}^{+0.31}$ & $-3.60 \pm 0.28$ \\
\hline & N-like & Si VIII & - & - & - & $-2.0 \pm 0.4$ \\
\hline & O-like & Si VII & - & - & - & - \\
\hline & H-like & S XVI & $-2.5 \pm 0.7$ & - & $-2.3 \pm 0.9$ & $-1.9 \pm 0.9$ \\
\hline & He-like & $\mathrm{S} \times \mathrm{V}$ & $-1.2 \pm 0.7$ & $-2.9 \pm 0.8$ & $-1.7 \pm 0.9$ & $-1.0 \pm 0.9$ \\
\hline & Li-like & S XIV & - & - & - & $-1.0 \pm 0.9$ \\
\hline & Be-like & S XIII & $-0.9 \pm 0.7$ & $-2.0 \pm 0.8$ & $-2.3 \pm 0.8$ & $-2.3 \pm 0.8$ \\
\hline & B-like & S XII & $-0.8 \pm 0.7$ & $-2.9 \pm 0.8$ & $-2.8 \pm 0.8$ & $-4.0 \pm 0.8$ \\
\hline & C-like & S XI & $-1.1 \pm 0.7$ & $-2.5_{-0.8}^{+0.9}$ & $-3.9 \pm 0.8$ & $-3.8 \pm 0.8$ \\
\hline & N-like & $\mathrm{Sx}$ & - & $-{ }^{-0.8}$ & $-2.3_{-0.8}^{+0.9}$ & $-2.7 \pm 0.9$ \\
\hline & O-like & S IX & - & - & $-{ }^{-0.8}$ & $-1.4 \pm 1.0$ \\
\hline
\end{tabular}




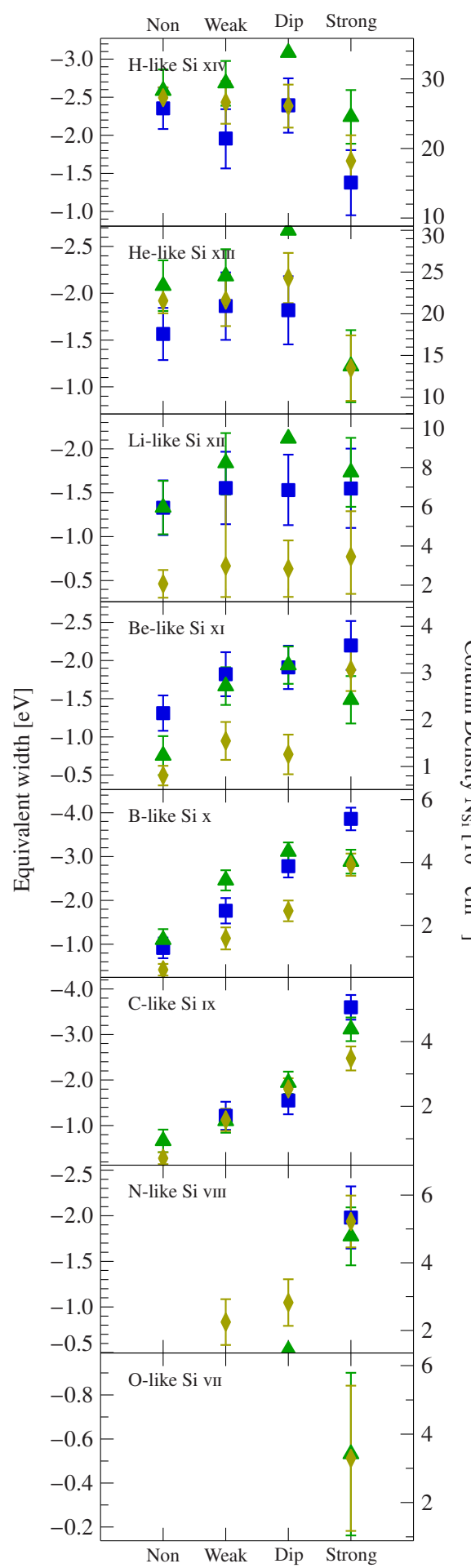

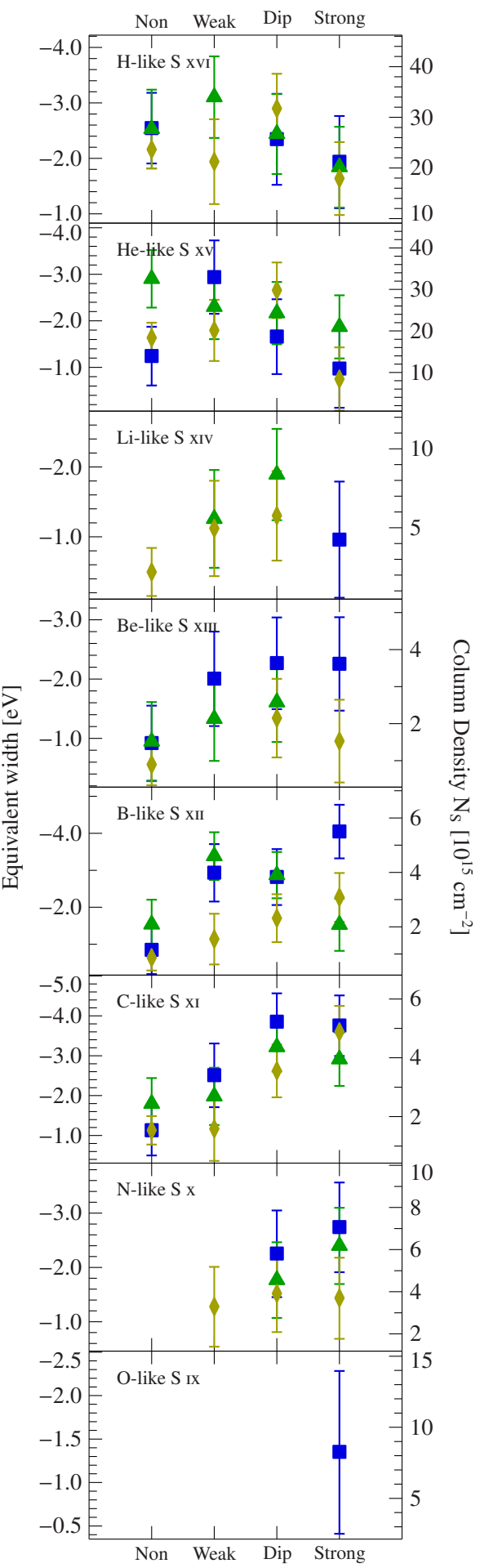

Fig. 6. Column densities for each dip stage derived from the respective equivalent width for all $\mathrm{Si}$ and $\mathrm{S}$ ion absorption lines detected in ObsIDs 3814 (yellow diamonds), 8525 (green triangles), and 9847 (blue squares). Negative values of the equivalent width correspond to absorption lines. The column densities of lower charged ions increase with increasing dip depth, whereas column densities of highly charged ions decrease with dip level. Be-like Si XI and Li-like Si XII lines are contaminated by the $\mathrm{Si} \mathrm{He} \mathrm{z} \mathrm{emission} \mathrm{line.}$
Figure 7 shows the column densities as a function of the ionization potential of the respective ions. The closed shell ions He-like Si XIII and S XV exhibit enhanced column densities as expected. However, whereas the appearance of lower ionization stages in the strong dip points to a lower temperature in the core of a clump, it is also possible that we see a constant temperature clump with a higher density in its core. As the absorber is ionized, the column density measured from continuum absorption is only a lower limit; we cannot see the fully ionized material. The figure also clearly illustrates the large column of the hydrogenic lines. It is therefore very likely that a significant amount of the $\mathrm{S}$ and $\mathrm{Si}$ in the system are fully ionized and thus cannot be detected (see also Sect. 3.1.3)

\subsubsection{Doppler shifts}

To analyze the morphology of the absorbing material, we next take a look at the bulk motion, measuring the Doppler shifts of the lines in the Si and S regions for each dipping stage. Since the uncertainty of the theoretical rest-wavelengths is on the order of the expected line shifts (Hell et al. 2013), we measured the centroids of the line blends for each ion in the laboratory using an electron beam ion trap and a microcalorimeter (Hell et al. 2016). 
Table 6. Column densities as calculated from the silicon line equivalent widths.

\begin{tabular}{|c|c|c|c|c|c|c|c|c|c|c|c|c|}
\hline & & $N_{\text {H-like }}$ & $N_{\text {He-like }}$ & $N_{\text {Li-like }}$ & $N_{\text {Be-like }}$ & $\begin{array}{l}N_{\text {B-like }} \\
\left.\mathrm{cm}^{-2}\right)\end{array}$ & $N_{\text {C-like }}$ & $N_{\mathrm{N}-\text { like }}$ & $N_{\text {O-like }}$ & $\begin{array}{l}N_{\mathrm{Si}} \\
\left(10^{15} \mathrm{~cm}^{-2}\right)\end{array}$ & $\begin{array}{l}N_{\mathrm{H}, \text { Wilms }} \\
\left(10^{21} \mathrm{~cm}^{-2}\right)\end{array}$ & $\begin{array}{l}N_{\mathrm{H}, \text { Herrero }}{ }^{(a)} \\
\left(10^{21} \mathrm{~cm}^{-2}\right)\end{array}$ \\
\hline \multirow{4}{*}{3814} & Non-dip & $54.6_{-3.1}^{+3.0}$ & $21.6 \pm 1.6$ & $2.1 \pm 0.8$ & $0.81 \pm 0.21$ & $0.59 \pm 0.19$ & $0.40 \pm 0.19$ & - & - & $80 \pm 7$ & $2.26 \pm 0.20$ & $0.89 \pm 0.08$ \\
\hline & Weak dip & $53 \pm 7^{-3.1}$ & $22 \pm 4$ & $3.0_{-1.6}^{+4.1}$ & $1.5 \pm 0.5$ & $1.6 \pm 0.4$ & $1.6 \pm 0.4$ & $2.2 \pm 0.7$ & - & $85 \pm 15$ & $2.4 \pm 0.5$ & $0.95 \pm 0.17$ \\
\hline & Dip & $52 \pm 7$ & $24 \pm 4$ & $2.8 \pm 1.5$ & $1.3 \pm 0.5$ & $2.5 \pm 0.4$ & $2.5 \pm 0.4$ & $2.8 \pm 0.7$ & - & $88 \pm 15$ & $2.5 \pm 0.4$ & $0.99 \pm 0.16$ \\
\hline & Strong dip & $36 \pm 8$ & $13 \pm 4$ & $3.5_{-1.9}^{+2.4}$ & $3.1 \pm 0.5$ & $3.9 \pm 0.4$ & $3.5 \pm 0.4$ & $5.2 \pm 0.8$ & $3.3 \pm 2.2$ & $72 \pm 18$ & $2.0 \pm 0.6$ & $0.81 \pm 0.21$ \\
\hline \multirow{4}{*}{8525} & Non-dip & $57 \pm 7$ & $23 \pm 4$ & $6.0 \pm 1.4$ & $1.2 \pm 0.5$ & $1.5 \pm 0.4$ & $0.9 \pm 0.4$ & - & - & $90 \pm 15$ & $2.5 \pm 0.4$ & $1.00 \pm 0.16$ \\
\hline & Weak dip & $59 \pm 7$ & $24 \pm 4$ & $8.2_{-1.7}^{+1.6}$ & $2.7 \pm 0.5$ & $3.4 \pm 0.4$ & $1.5 \pm 0.4$ & - & - & $99 \pm 15$ & $2.8 \pm 0.5$ & $1.11 \pm 0.17$ \\
\hline & Dip & $68 \pm 7$ & $29 \pm 4$ & $9.5_{-1.4}^{+1.1}$ & $3.2_{-0.5}^{+0.4}$ & $4.35_{-0.31}^{+0.30}$ & $2.7 \pm 0.4$ & $1.4 \pm 0.8$ & - & $119 \pm 15$ & $3.3 \pm 0.4$ & $1.32 \pm 0.16$ \\
\hline & Strong dip & $49 \pm 8$ & $13 \pm 5$ & $7.8 \pm 1.8$ & $2.4 \pm 0.6$ & $4.0 \pm 0.4$ & $4.4 \pm 0.4$ & $4.8 \pm 0.9$ & $3.4 \pm 2.4$ & $90 \pm 19$ & $2.5 \pm 0.6$ & $1.00 \pm 0.21$ \\
\hline \multirow{4}{*}{9847} & Nnon-dip & $52 \pm 6$ & $18 \pm 4$ & $5.9 \pm 1.4$ & $2.1 \pm 0.4$ & $1.3 \pm 0.4$ & - & - & - & $78 \pm 14$ & $2.2 \pm 0.4$ & $0.88 \pm 0.16$ \\
\hline & Weak dip & $43 \pm 9$ & $21 \pm 5$ & $7.0 \pm 1.9$ & $3.0 \pm 0.5$ & $2.5 \pm 0.5$ & $1.7 \pm 0.5$ & - & - & $78 \pm 20$ & $2.2 \pm 0.6$ & $0.87 \pm 0.22$ \\
\hline & Dip & $52 \pm 8$ & $20 \pm 5$ & $6.8_{-1.8}^{+1.9}$ & $3.1 \pm 0.5$ & $3.9 \pm 0.4$ & $2.2 \pm 0.5$ & - & - & $89 \pm 19$ & $2.5 \pm 0.6$ & $0.99 \pm 0.21$ \\
\hline & Strong dip & $30 \pm 10$ & - & $6.9 \pm 2.1$ & $3.6 \pm 0.6$ & $5.4 \pm 0.4$ & $5.1 \pm 0.4$ & $5.3 \pm 1.0$ & - & $57 \pm 20$ & $1.6 \pm 0.6$ & $0.63 \pm 0.22$ \\
\hline
\end{tabular}

Notes. ${ }^{(a)}$ Herrero et al. (1995) provide a scaling factor for the observed overabundance of helium, which we apply to the solar abundances of Wilms et al. (2000). See text for further details.

Table 7. Column densities as calculated from the sulfur line equivalent widths.

\begin{tabular}{|c|c|c|c|c|c|c|c|c|c|c|c|c|}
\hline & & $N_{\text {H-like }}$ & $N_{\text {He-like }}$ & $N_{\text {Li-like }}$ & $\begin{array}{l}N_{\text {Be-like }} \\
\quad\left(10^{15}\right.\end{array}$ & $\begin{array}{l}N_{\text {B-like }} \\
\left.\mathrm{cm}^{-2}\right)\end{array}$ & $N_{\text {C-like }}$ & $N_{\mathrm{N}-\text { like }}$ & $N_{\text {O-like }}$ & $\begin{array}{l}N_{\mathrm{S}} \\
\left(10^{15} \mathrm{~cm}^{-2}\right)\end{array}$ & $\begin{array}{l}N_{\mathrm{H}, \text { Wilms }} \\
\left(10^{21} \mathrm{~cm}^{-2}\right)\end{array}$ & $\begin{array}{l}N_{\mathrm{H}, \text { Herrero }}{ }^{(a)} \\
\left(10^{21} \mathrm{~cm}^{-2}\right)\end{array}$ \\
\hline \multirow{4}{*}{3814} & Non-dip & $47 \pm 8$ & $18 \pm 4$ & $2.2 \pm 1.6$ & $0.9 \pm 0.6$ & $0.9 \pm 0.5$ & $1.5 \pm 0.5$ & - & - & $71 \pm 18$ & $2.0 \pm 0.5$ & $0.79 \pm 0.19$ \\
\hline & Weak dip & $43 \pm 17$ & $20 \pm 8$ & $5 \pm 4$ & - & $1.6 \pm 1.0$ & $1.6 \pm 1.1$ & $3.3_{-2.0}^{+1.9}$ & - & $70 \pm 40$ & $2.1 \pm 1.1$ & $0.8 \pm 0.5$ \\
\hline & Dip & $64 \pm 14$ & $30 \pm 7$ & $5.8 \pm 2.9$ & $2.2 \pm 1.1$ & $2.3 \pm 0.9$ & $3.6_{-1.0}^{+0.9}$ & $3.9 \pm 1.9$ & - & $110 \pm 40$ & $3.1 \pm 0.9$ & $1.2 \pm 0.4$ \\
\hline & Strong dip & $36 \pm 15$ & $8 \pm 8$ & - & $1.5 \pm 1.2$ & $3.1_{-1.0}^{+0.9}$ & $4.9 \pm 0.9$ & $3.7 \pm 2.0$ & - & $60 \pm 40$ & $1.6 \pm 1.0$ & $0.6 \pm 0.4$ \\
\hline \multirow{4}{*}{8525} & Non-dip & $55 \pm 16$ & $33 \pm 7$ & - & $1.5 \pm 1.1$ & $2.1 \pm 1.0$ & $2.4 \pm 0.9$ & - & - & $90 \pm 40$ & $2.8 \pm 1.0$ & $1.0 \pm 0.4$ \\
\hline & Weak dip & $68 \pm 17$ & $26 \pm 8$ & $6 \pm 4$ & $2.1 \pm 1.2$ & $4.6 \pm 0.9$ & $2.7 \pm 1.0$ & - & - & $110 \pm 40$ & $3.1 \pm 1.1$ & $1.2 \pm 0.5$ \\
\hline & Dip & $53 \pm 16$ & $24 \pm 8$ & $8.4_{-3.0}^{+2.9}$ & $2.6 \pm 1.1$ & $3.9 \pm 0.9$ & $4.4 \pm 0.9$ & $4.6_{-1.9}^{+1.8}$ & - & $100 \pm 40$ & $2.9 \pm 1.1$ & $1.1 \pm 0.4$ \\
\hline & Strong dip & $40_{-17}^{+16}$ & $21 \pm 8$ & - & - & $2.1 \pm 1.0$ & $4.0 \pm 1.0$ & $6.2_{-1.9}^{+1.8}$ & - & $70 \pm 40$ & $2.1 \pm 1.1$ & $0.8 \pm 0.4$ \\
\hline \multirow{4}{*}{9847} & Non-dip & $56 \pm 14$ & $14 \pm 8$ & - & $1.5 \pm 1.1$ & $1.2 \pm 0.9$ & $1.5 \pm 0.9$ & - & - & $70 \pm 40$ & $2.1 \pm 0.9$ & $0.8 \pm 0.4$ \\
\hline & Weak dip & - & $33 \pm 9$ & - & $3.2 \pm 1.3$ & $4.0 \pm 1.1$ & $3.4 \pm 1.1$ & - & - & $44 \pm 19$ & $1.2 \pm 0.6$ & $0.49 \pm 0.21$ \\
\hline & Dip & $51_{-19}^{+18}$ & $19_{-10}^{+9}$ & - & $3.6 \pm 1.3$ & $3.8 \pm 1.1$ & $5.2 \pm 1.0$ & $5.8 \pm 2.1$ & - & $90 \pm 50$ & $2.5 \pm 1.2$ & $1.0 \pm 0.5$ \\
\hline & Strong dip & $42 \pm 19$ & $11 \pm 10$ & $4 \pm 4$ & $3.6 \pm 1.3$ & $5.5 \pm 1.0$ & $5.1 \pm 1.1$ & $7.1 \pm 2.2$ & $8 \pm 6$ & $90 \pm 50$ & $2.5 \pm 1.3$ & $1.0 \pm 0.5$ \\
\hline
\end{tabular}

Notes. ${ }^{(a)}$ Herrero et al. (1995) provide a scaling factor for the observed overabundance of helium, which we apply to the solar abundances of Wilms et al. (2000). See text for further details.

For the shifts of the He- and H-like $1 \mathrm{~s} \rightarrow 2 \mathrm{p}$ transitions, we used the tables of Drake (1988) and Garcia \& Mack (1965), respectively, as reference. These are the same reference publications that we also used for the calibration of the laboratory data.

Figure 8 shows the resulting velocities for each line in the different dipping stages of each observation. Overall, for the same spectral lines the Doppler shifts are consistent throughout the different dipping stages. This is more obvious for the uncontaminated lines in the Si spectra, where statistics are much better than for the $\mathrm{S}$ lines and line centers can be determined more accurately. The O-like S IX line detected in ObsID 9847 is the only outlier. As the width of this line is narrow (only one energy bin), and since we only claim a tentative detection of this line (see Sect. 3.1), the derived Doppler velocity is probably inaccurate and we do not include the line in Fig. 8.

Figure 9 shows the distribution of the Doppler shifts in the three observations. For ObsID 3814, the velocities scatter around $0 \mathrm{~km} \mathrm{~s}^{-1}$. For ObsID 8525, the velocities increase toward negative values and scatter around $-60 \mathrm{~km} \mathrm{~s}^{-1}$, and for ObsID 9847 , the scatter moves down to around $-230 \mathrm{~km} \mathrm{~s}^{-1}$.
This Doppler shift cannot be due to the free fall velocity of donor material onto the black hole. As we look at the system near $\phi_{\text {orb }}=0$, the free fall velocity would cause a redshift, not a blueshift of lines as seen in the spectra. The Keplerian velocity of the black hole cannot be the cause for the energy shift either, as we would expect the maximum amplitude of the shift to be on the order of $100 \mathrm{~km} \mathrm{~s}^{-1}$ and not much larger than that; the projected semi-amplitude of the black hole is $K_{\mathrm{BH}} \sim 91 \mathrm{~km} \mathrm{~s}^{-1}$ (e.g., Brocksopp et al. 1999; Gies et al. 2003; Orosz et al. 2011).

\subsubsection{A clumpy absorber}

The consistency of the Doppler shifts of the lines during dipping provides evidence for inhomogeneities in the absorber that are forming a unified structure that has some kind of density stratification. We call these structures clumps, even though we do not want to imply that the structures are static phenomena as opposed to transient structures. Either owing to self shielding, or because the material is in some kind of approximate pressure equilibrium similar to the clouds in active galaxies 

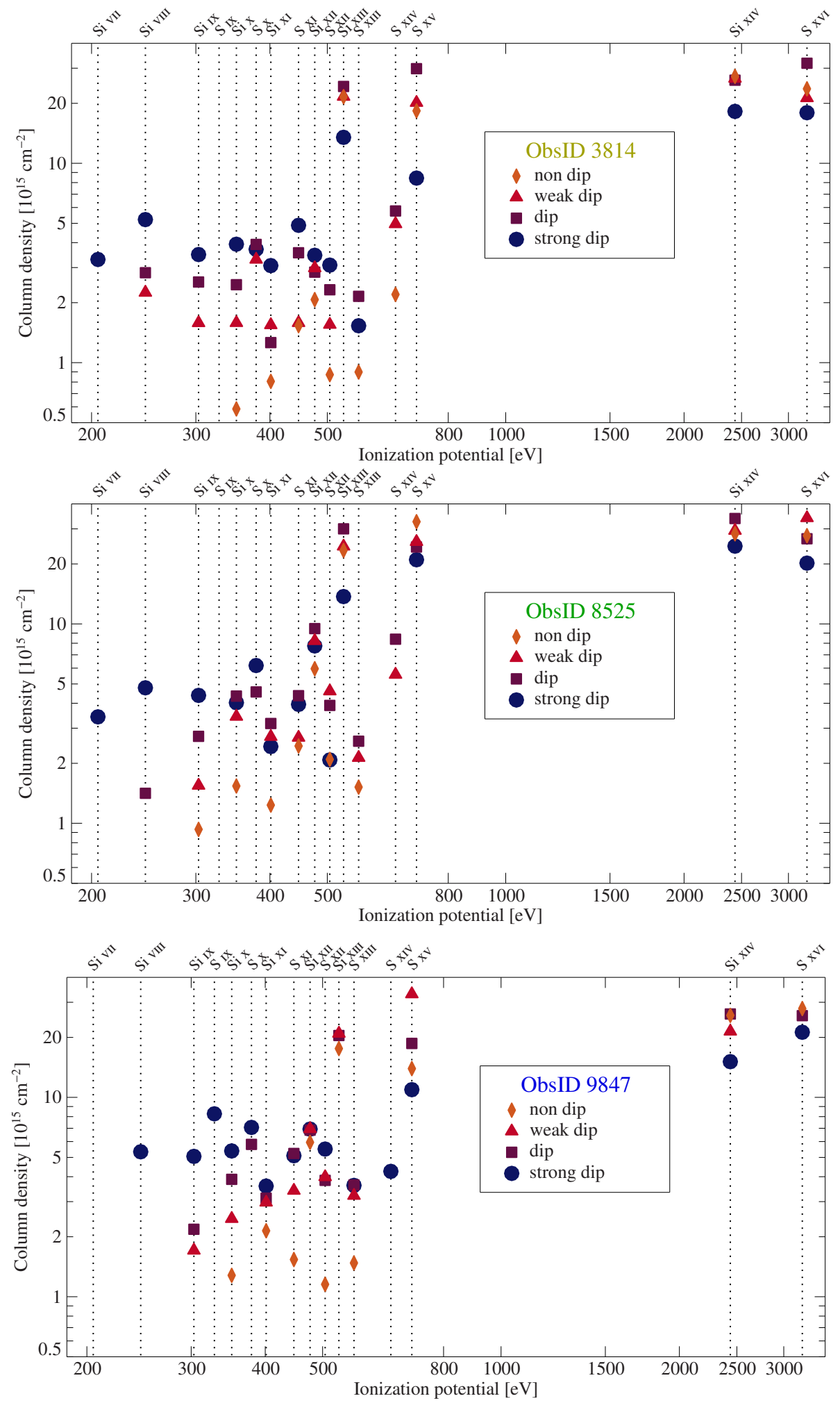

Fig. 7. Column densities vs. ionization potential for the respective silicon and sulfur ions in all dip stages.

(Krolik et al. 1981), the part of the clump facing away from the black hole is less ionized. As the clump moves through the line of sight (Fig. 10), we first look through its outer, more ionized regions. We only see the lower ionization stages during the deeper dip where the middle of the clump is located in our line of sight.

Regarding the orbital phase dependency, from the three ObsIDs analyzed we can already see a trend toward higher Doppler velocities further away from $\phi_{\text {orb }} \sim 0$. This is consistent with Paper II. Figure 11 shows the median, mean, first, and third quartile of the velocity distribution of the Si and $\mathrm{S}$ lines in comparison to the non-dip results shown in Fig. 11 of Paper II. The phase dependence of the Doppler shifts measured within the dips as well as the amplitude are roughly consistent with Paper II. The material producing the dips thus seems to be at the same distance from the black hole as what can be seen during non-dip.

The projected wind velocities in this figure are calculated using a CAK-model with a focused wind based on 


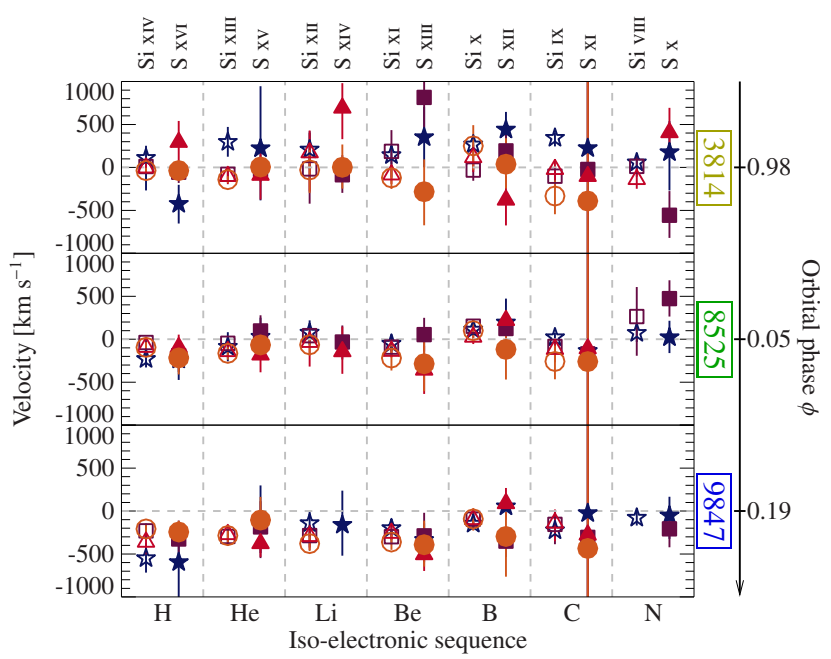

Fig. 8. Doppler velocities for the various ionization states of Si VIIISi XIV (empty) and S X-S XVI (filled) in all dipping stages (non-dip: orange circles; weak dip: red triangles; dip: violet squares; and strong dip: blue stars) over all observations in the different orbital phases.

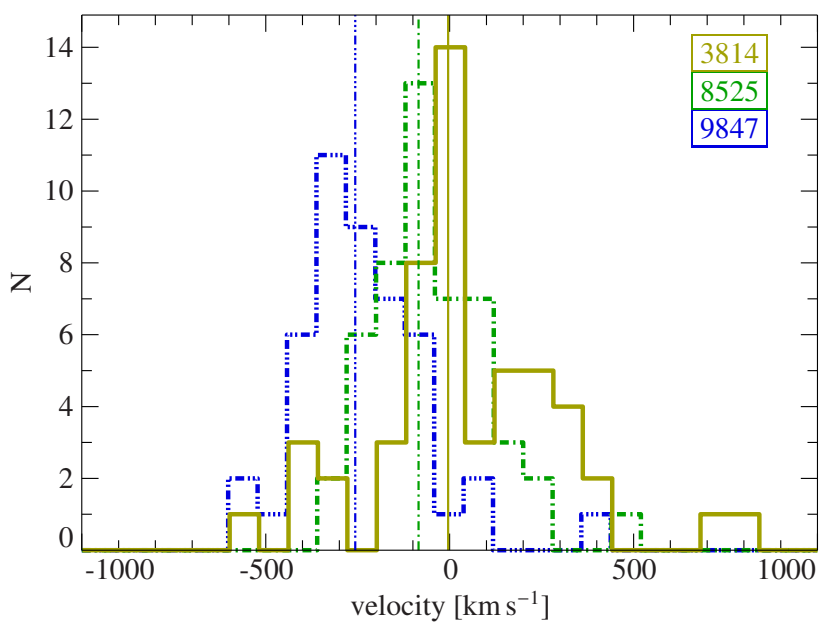

Fig. 9. Distribution of velocity shifts of all $\mathrm{Si}$ and $\mathrm{S}$ lines detected in the three ObsIDs with their medians. The peak velocity clearly shifts between the observations.

Gies \& Bolton (1986b, see Paper II for more details ${ }^{6}$ ). The model consists of a radially symmetric wind that is focused onto the black hole in a cone of $20^{\circ}$. Gies \& Bolton (1986b) stated that their model is valid only out to $0.9 d$ away from the stellar center, where $d$ is the distance between the star and the black hole, as the density at this distance is already too small to produce a significant contribution to the absorption line profiles. It is possible to argue that this is only the case for a smooth wind and clumps have to be treated differently. Even for $\phi_{\text {orb }}=0$ the regions we are interested in (distances of $0-0.75 d$ from the black hole, in the direction of the line of sight at different phases) are mostly outside of the valid region of the model of Gies \& Bolton (1986b), but as our observations are near $\phi_{\text {orb }}=0$, where the distance to the star is not much more than $0.9 d$, we still take the model for a rough estimate. However, both measured non-dip and dip Doppler shifts fit neither the projected wind velocity

\footnotetext{
6 There was a sign error in the code that produced the figure in Paper II. This led to a flip of sign for the velocity curves and a slight phase shift. These changes do not have an influence on the interpretation of the figure in Paper II. Figure 11 shows the corrected velocity curves.
}

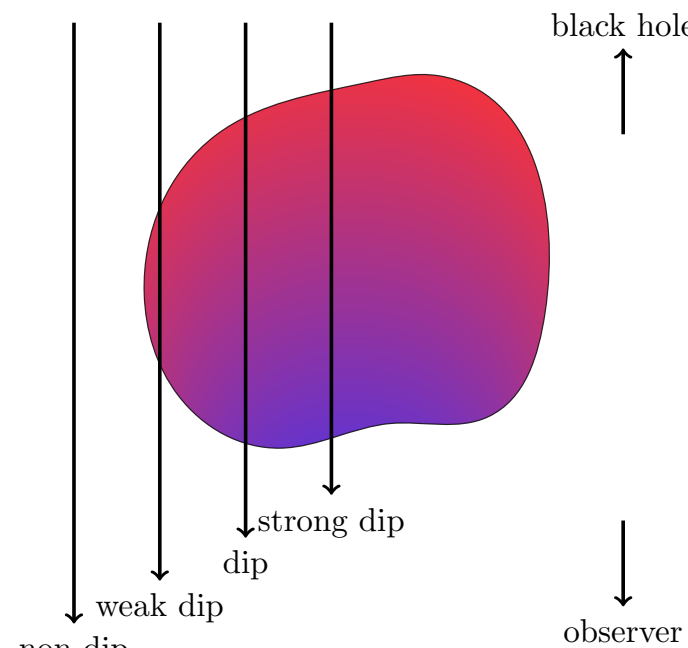

Fig. 10. Sketch of a clump passing our line of sight and causing the different dip stages. Red color indicates more highly ionized regions; blue color regions indicate lower ionization balance.

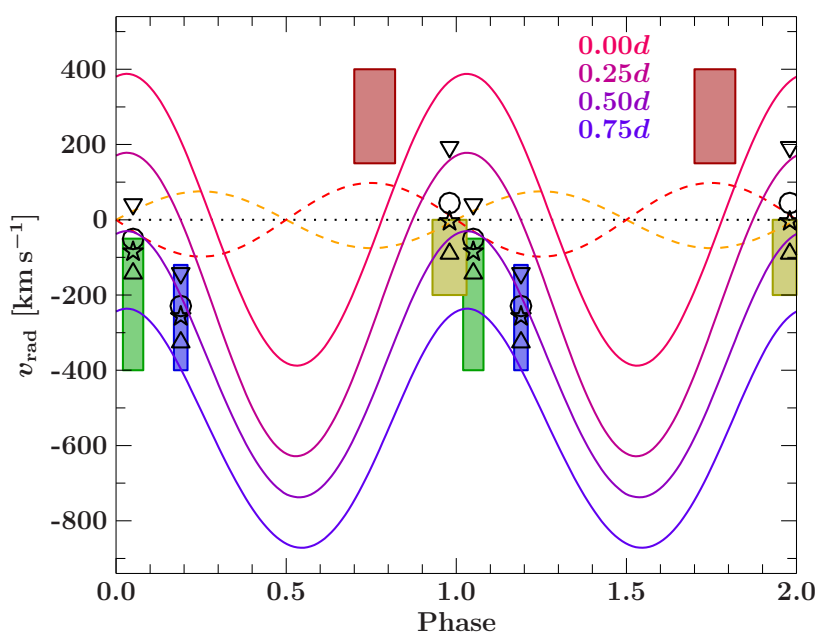

Fig. 11. Radial velocities of the black hole (red dashed line) and the companion (orange dashed line), and wind velocities projected onto the line of sight as a function of distance from the black hole. Distances are given in units of the distance between the black hole and its donor, $d$. Colored boxes indicate the range of measured non-dip Doppler shifts from the Chandra observations (red: ObsID 3815; yellow: ObsID 3814; green: ObsID 8525; and blue: ObsID 9847, modified and corrected from Paper II, Fig. 11). Symbols show the median (star), mean (circle), first (point-up triangle), and third quartile (point-down triangle) of the distribution of Doppler shifts for the Si and S lines.

(difference in phase) nor the black hole (difference in amplitude). Thus, we cannot make an exact statement on the location of the clumps in the system from our data, although a comparison of the amplitude implies a distance of less than $0.25 d$ from the black hole.

In order to investigate the origin of the observed ionization, we use XSTAR (Kallman \& Bautista 2001), which models the photoionization structure of spherically symmetric gas clouds that are irradiated by a central source of arbitrary spectral energy distribution. We assume gas clouds with the default elemental composition used in XSTAR, which is based on Grevesse et al. (1996). The clouds have a covering fraction of $100 \%$, a temperature of $10^{6} \mathrm{~K}$, a constant total hydrogen particle density of $n=10^{10} \mathrm{~cm}^{-3}$, and are irradiated by an incident $1-1000 \mathrm{Ry}$ 


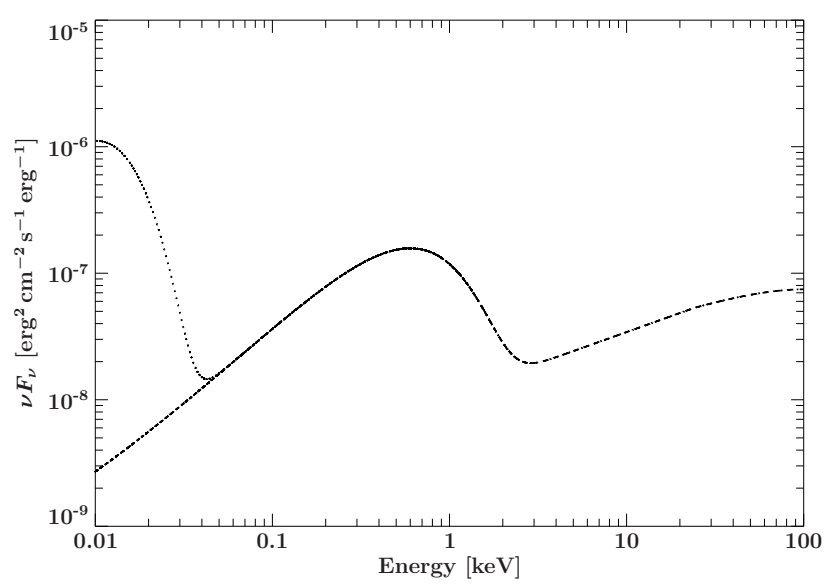

Fig. 12. Incident SED used for the XSTAR simulations. The dotted and dashed lines show the SED with and without the optical companion star, respectively. We note that the SED is always renormalized to the assumed model luminosity.

luminosity of $L=1.4 \times 10^{37} \mathrm{erg} \mathrm{s}^{-1}$. In this configuration, the definition of the ionization parameter is $\xi=L /\left(n R^{2}\right)$, where $R$ is the inner radius of the shell. Our incident spectral energy density (SED) is adapted from Pepe et al. (2015) and assumes that the source of X-rays is point like. Any mechanical input from the jet in the system is ignored. In order to gauge the influence of the strong UV photon field of the donor star, we perform simulations with and without a stellar contribution to the total SED (see Fig. 12).

We calculate model grids by varying $\xi$ and the total hydrogen column, $N_{\mathrm{H}}=n \ell$, where $\ell$ is the geometric size of the cloud. Since $L$ and $n$ are fixed, this approach effectively models photoionized clouds of varying sizes and distances from the source of ionizing photons. The left panel of Fig. 13 shows the columns for the ions of $\mathrm{Si}$ and $\mathrm{S}$ as a function of $\log \xi$ for $N_{\mathrm{H}}=10^{22} \mathrm{~cm}^{-2}$. There is no single value of $\log \xi$ that produces the measured columns for all ions under consideration. This result holds for all values of $N_{\mathrm{H}}$. Based on a grid of XSTAR simulations in which we vary $\log \xi$ and $N_{\mathrm{H}}$, in the right-hand panel of Fig. 13, we show the contours in the $\log \xi-N_{\mathrm{H}}$-plane in which the theoretical column of each ion of $\mathrm{Si}$ and $\mathrm{S}$ ion equals that measured during the strong dip of observation 8525. In the ideal case of a simple medium of constant density and temperature, all of these contours would intersect in a single point; the modulo measurement errors are, however, small compared to the large dynamic range shown in Fig. 13. We note that the inclusion of the optical companion has only a marginal effect on the observed ion column densities of $\mathrm{Si}$ and $\mathrm{S}$ (Fig. 13, right, panels $\mathrm{c}$ and d). This is important because the binary geometry of the system with two sources of radiation cannot be accounted for by the XSTAR simulation. Neglecting the optical companion therefore only introduces a small systematic effect on ionization structure modeling of these ions.

In practice, assuming that the dip is produced by a single absorbing cloud, we would expect some kind of a density variation in the cloud, as discussed above. We can use our simple XSTAR simulations to identify regions of particular interest. From Fig. 13, columns of moderately ionized ions can be roughly reproduced with hydrogen column densities around $3 \times 10^{20} \mathrm{~cm}^{-2}$ and ionization parameters of $\log \xi \sim 1-2$. The high ionization states of silicon and sulfur, on the other hand, require column densities and an ionization parameter that are roughly an order of magnitude higher. This result points at a more complex origin for the ionization structure than photoionization alone. One possible explanation would be ionization due to the strong shocks that are present in hydrodynamic simulations for massive X-ray binary systems (Blondin et al. 1990, 1991; Blondin 1994; Manousakis \& Walter 2011, 2015; Sundqvist et al. 2018, and references therein) and in winds from early-type stars (Owocki et al. 1988; Sundqvist \& Owocki 2013, and references therein).

\subsubsection{Line symmetry}

Although a detailed analysis of the complex photoionization line profiles observed in Cyg X-1 is beyond the scope of this paper, we can at least take a quantitative look at the line shapes in the region of \pm 8 bins around the respective rest energy, adjusted for the most probable Doppler shift for each ObsID (3814: $0 \mathrm{~km} \mathrm{~s}^{-1}$, 8525: $-80 \mathrm{~km} \mathrm{~s}^{-1}, 9847:-320 \mathrm{~km} \mathrm{~s}^{-1}$; see also Fig. 9). Figure 14 shows the original data (colored) and the same data flipped (black) around the center of the bin containing the adjusted rest energy (gray dashed line). For a symmetric line shape, the original and flipped data should match, as is the case, for example, for $8525 \mathrm{He}$-like Si XIII in the dip data, or $3814 \mathrm{H}$-like Si XIV non-dip, weak dip and dip data. However, often we see the lines shifted by one or even two bins (e.g., 3814 C-like Si IX strong dip or 9847 B-like S XII weak dip, dip and strong dip). There are also signs of asymmetry (e.g., 9847 C-like Si IX strong dip or 3814 C-like S XI dip), as well as lines that show both asymmetry and a shift (e.g., 3814 C-like Si IX strong dip or 8525 B-like Si X non-dip and dip).

Clear P Cygni-like profiles for Cyg X-1 were reported only at $\phi_{\text {orb }}=0.5$ during ObsID 11044 (Miškovičová et al. 2011). Schulz et al. (2002a) also found indications of P Cygni profiles at $\phi_{\text {orb }}=0.74$. In the UV, P Cygni profiles were found by Vrtilek et al. (2008), also at $\phi_{\text {orb }}=0.5$, where the stellar wind is focused toward the observer by the black hole. At around $\phi_{\text {orb }}=0$, the focused wind is moving away from the observer toward the black hole, and the redshift and ionization are highest. Asymmetries in the lines are thus expected at that phase. This was observed, for example, by Feng et al. (2003) and discussed in Paper II. Our observations fit this picture.

\subsection{Emission in the deepest dips}

While the absorption lines discussed so far show a clear dependency on the depth of the dip, this is not the case for the He-like Si XIII z emission line, whose total line flux is constant throughout the dip stages. This result could either indicate that the emitting material originates in a geometrically much larger region than the absorber, such that the emission line is not affected by the absorber, or, alternatively, that the emission line originates in an area where the line of sight does not pass through the absorber.

A possible origin for the Si XIII emission line could be the photoionization region in the stellar wind that surrounds the X-ray emission region. As discussed in Paper I, the high ionization region around the $\mathrm{X}$-ray source, similar to the Strömgren sphere around stars, is comparable in size to the separation of the black hole and its donor star, i.e., it is much larger than the absorbing cloud. Given the low optical depth of this region we expect a region whose $\mathrm{X}$-ray emission is dominated by emission lines, similar to that seen, for example, in Vela X-1 (Schulz et al. 2002b; Watanabe et al. 2006; Grinberg et al. 2017) or in Cen X-3 (Wojdowski et al. 2003). The flux from the photoionization region, however, is much fainter than the X-rays from the accretion flow. The region has therefore been mainly 

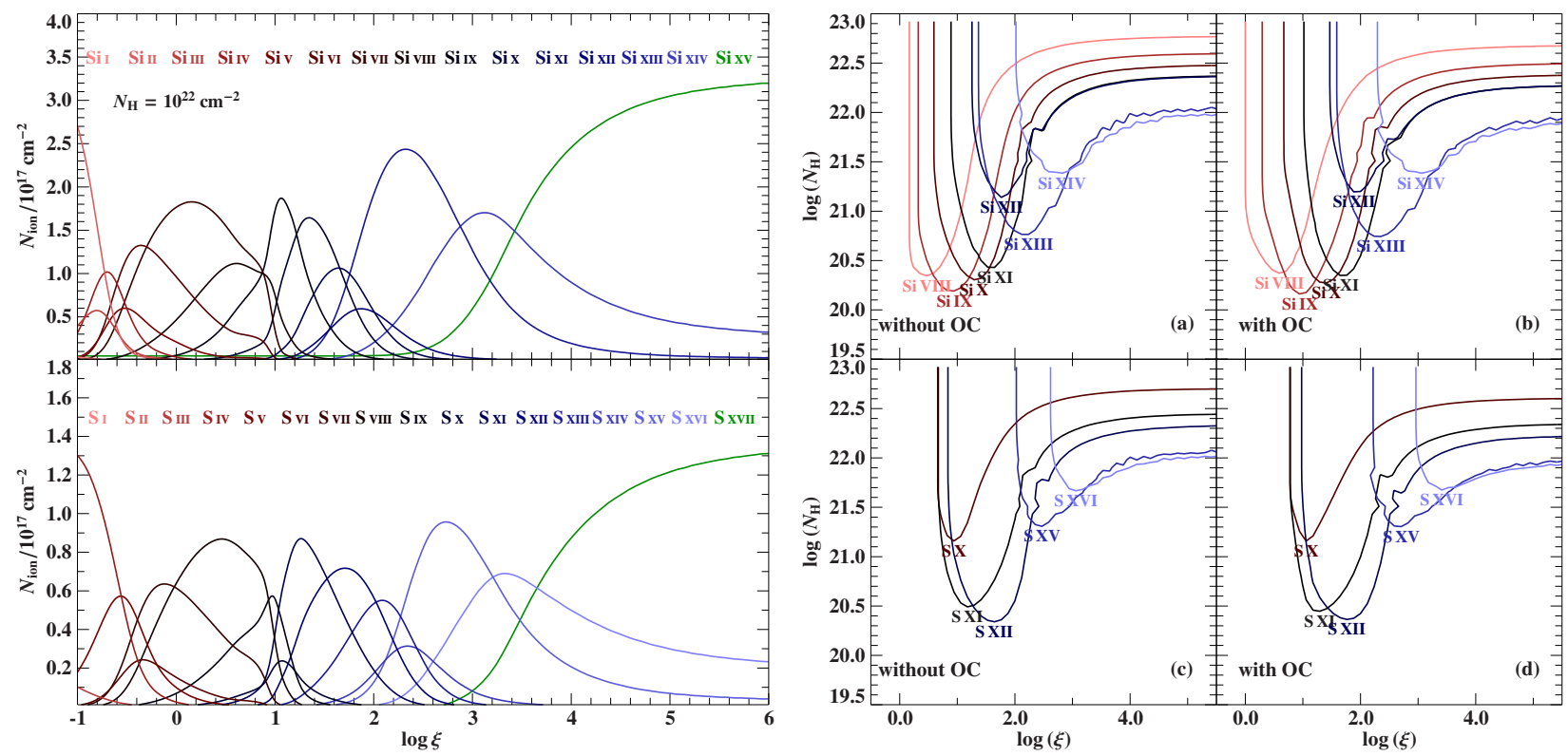

Fig. 13. Left panel: predicted columns for the ions of $\mathrm{Si}$ and $\mathrm{S}$ for a spherical cloud with $N_{\mathrm{H}}=10^{22} \mathrm{~cm}^{-2}$ that is irradiated by a X-ray source with the spectrum defined by Fig. 12. Right panel: lines of constant ion column density color-coded for each different ion of Si (panels a and $b$ ) and $\mathrm{S}$ (panels $c$ and $d$ ), for XSTAR simulated values of equivalent hydrogen column density, $N_{\mathrm{H}}$, and ionization parameter, $\log \xi$. The assumed ion columns are the values measured during the strong dip of observation 8525 (Table 5). If a simple XSTAR model described the observation, we would expect these lines to cross in one point (within their error bars). The left column of the figure shows calculations without the effect of the optical companion (OC); the right column includes the UV radiation from the companion.

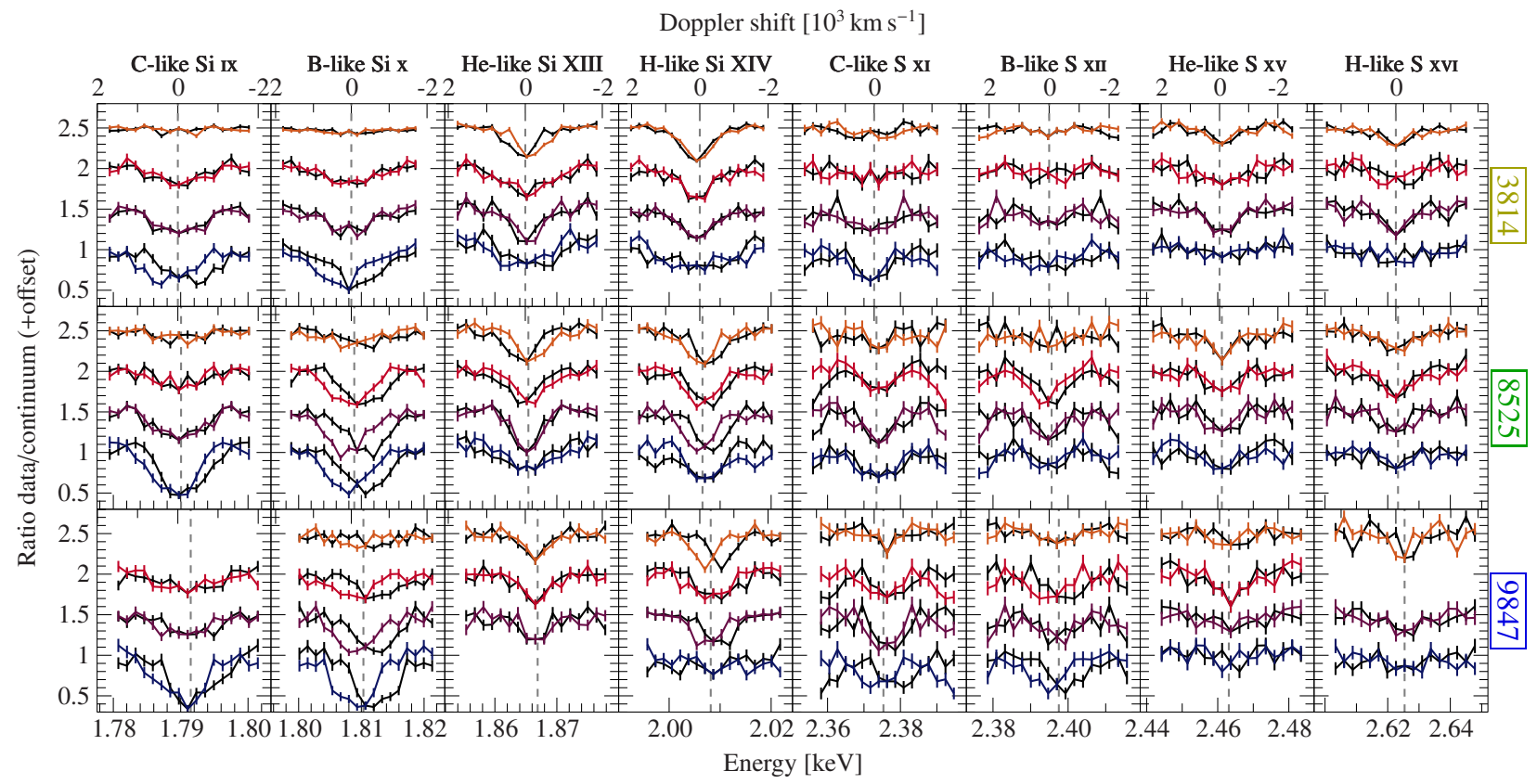

Fig. 14. Original line profiles (colored) for the non-dip (top, orange), weak dip (second from top, red), dip (third from top, purple), and strong dip (bottom, blue) lines for selected ions, as well as the same data flipped (black, see also text for further information) at the energy corresponding to adding a constant Doppler shift for each observation (vertical dashed line) to the line's laboratory energy. For an ideal, symmetric line, the colored and black curves should match. Most of the lines observed have complex line profiles.

studied for edge-on systems, where our line of sight onto the bright X-ray source is blocked by the donor star during eclipses. While the Cyg X-1/HDE 226868 system does not show eclipses, we can utilize the strong dipping to at least partially block the line of sight to the black hole. The best opportunity for this is presented by the long, deep dip at the beginning ObsID 8525 , which is the most pronounced of all the dips in our observations. The deep part of this dip (according to our dip stage identifica- tion, i.e., the blue data points in Fig. 1 belonging to that dip) lasted for $5.3 \mathrm{ks}(1.5 \mathrm{~h})$, which is long enough to accumulate a decent gratings spectrum of one single dip. Because of the strong absorption, pileup is no concern in the resulting spectrum and the HEG and MEG spectra agree with each other.

As shown in Fig. 15, during the deepest parts of the dip in ObsID 8525, the photoionized zone around the black hole indeed dominates the spectrum, resulting in the presence of 


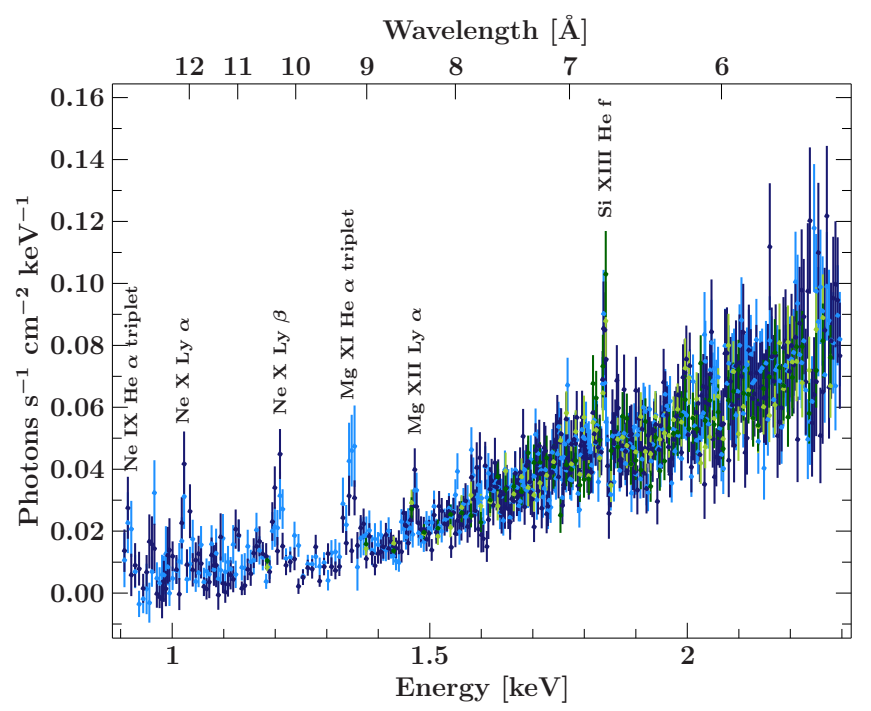

Fig. 15. Deep dip spectrum from ObsID 8525 from a subset of the strong dip data in the $0.9-2.3 \mathrm{keV}(13.8-5.4 \AA)$ range. The data are rebinned to $S / N \geq 5$, revealing emission lines from the photoionized zone around the black hole.

emission lines. The most prominent lines detected are Si XIII He z (1.8394 keV $\equiv 6.7405$; Porquet et al. 2010), Mg Ly $\alpha$ $(1.4723 \mathrm{keV} \equiv 8.4211 \AA)$, the $\operatorname{MgHe} \alpha$ triplet $(\sim 1.3522 \mathrm{keV} \equiv$ $9.1691 \AA)$, as well as Ne Ly $\beta(1.2109 \mathrm{keV} \equiv 10.2390 \AA)$, Ne Ly $\alpha$ $(1.0280 \mathrm{keV} \equiv 12.0601 \AA)$, and the Ne IX He $\alpha$ triplet $(\sim 0.9220 \mathrm{keV} \equiv 13.4473 \AA)$. Unfortunately, however, the signal to noise in the lines is too low to allow a further characterization of their parameters.

\section{Summary}

We have analyzed three Chandra-HETGS observations of Cyg X-1 around superior conjunction of the system, where the structure of the stellar wind causes absorption features, so-called dips, in the X-ray light curve. With the help of color-color diagrams, we divide the data into four different dip stages containing a comparable total number of counts. For each dip stage, we fit the silicon and sulfur regions of the spectrum by applying a local power law continuum and Gaussian lines to model the absorption lines for different ionization stages of silicon and sulfur. We find lower charge ions appearing in the deeper dip stages, equivalent widths/absorption columns rising for deeper dip stages, phase dependent Doppler shifts for the lines, asymmetric line shapes, and emission lines emerging in the deepest dip.

The lower charge states of both elements only appear in the deeper dip stages, pointing toward colder material shielded from the irradiation by the black hole. The total columns in the dip stages derived from the equivalent widths of the lines represent lower limits to the real values, as we cannot see the fully ionized material adding to the column, which is likely to carry most of the mass. The derived $N_{\mathrm{H}}$ values of $N_{\mathrm{H}} \sim 2-4 \times 10^{21} \mathrm{~cm}^{-2}$ are in agreement with previous results from Paper I, although there are slight differences due to differences in the continuum.

The Doppler shifts of the lines with respect to measured laboratory values (Hell et al. 2016) show a scatter between the different dip stages, but the same trend within a single observation, pointing toward a single structure containing different ionization stages, which moves as a whole with a certain speed. The Doppler velocities show a clear modulation with orbital phase and are in agreement with the results found in Paper II. However, these velocities match neither the expected wind velocities calculated from a toy model after Gies \& Bolton (1986a) nor free fall nor the Kepler velocity around the black hole. Thus, from the line shifts it is not possible to tell where the material causing the absorption dips in the light curve is located.

For the three observations around $\phi_{\text {orb }}=0$, the lines show asymmetries. This is expected from previous observations near superior conjunction (see Paper I and references therein). Clear P Cygni-like profiles are reported only near $\phi_{\text {orb }}=0.5$.

Within the deepest dip, there is an emission line spectrum emerging that is outshone otherwise. Together with the Si XIII emission line that does not change its total line flux during the dip stages, this emission line spectrum indicates a much larger area responsible for the emission than what is absorbed by the wind structures, or an area distant from the absorber such that the line of sight toward it is not affected. A possibility is the almost fully ionized photoionization region in the stellar wind surrounding the X-ray emission region.

\section{Conclusions}

Our analysis gives us a glimpse at the properties of the structures causing the dips. From the light curve, we can constrain the duration of a dip, and thus of a passage of the clump through our line of sight, from $0.5 \mathrm{ks}$ for the shorter dips up to $5 \mathrm{ks}$ for the longer dips. This assumption is only valid if a dip is caused by a single, bigger clump with a cold or dense core instead of many small clumps in the line of sight.

Assuming a distance of $0.25 d$ between the clump and the black hole ( $d$ being the distance between black hole and donor; see Sect. 3.1.2), a simple Keplerian approach (i.e., the clump orbiting the black hole) gives a velocity of $\sim 460 \mathrm{~km} \mathrm{~s}^{-1}$ perpendicular to the line of sight. From the velocity of the clump and the duration of the dip, we can estimate the diameter of the clumps to $(2.3-23) \times 10^{8} \mathrm{~m}$, equivalent to $(0.33-3.3) R_{\odot}$ or $(0.02-0.2) R_{*}$. As an advancing clump grows in size (Sundqvist et al. 2012), we can calculate its ejection size (Grinberg et al. 2015) to about a third of the size that we measure at the distance of $0.25 \mathrm{~d}$.

With the current data we can only speculate whether this picture of single, big clumps is the correct one. The morphology of the light curve in ObsID 8525 supports this idea, whereas ObsID 3814 with its rapid variability appears to be more in favor of a large number of small clumps in our line of sight. For a sufficiently high number of smaller clumps, those closer to the black hole are partly shielding the outer ones from the ionizing radiation, which is in agreement with our observation of lower ionization stages during strong dipping. A possible explanation would then be a mixture of some larger clumps, causing the distinct dips in the light curve, and many smaller clumps.

This idea is consistent with the picture of structured, radiation pressure driven O-star winds (Castor et al. 1975). As shown, for example, by Owocki \& Rybicki (1984), the stationary solution for a line-driven wind is unstable. These instabilities grow quickly and result in strong shocks. Nonstationary hydrodynamic simulations show that dense cool shells of gas already form in deep wind regions close to the photosphere of the O star (Feldmeier et al. 1997; Dessart \& Owocki 2003; Oskinova et al. 2012; Sundqvist et al. 2018). Large density, velocity, and temperature variations due to the de-shadowing instability further compress the gas in these shells and fragment these shells into clumps. Theory predicts thus that the wind is actually a two-phase medium consisting of tenuous, hot gas (covering most of the volume) and embedded cool, dense clumps (containing 
most of the mass). Observational evidence for this clumping has been found for isolated O-type supergiants (e.g., Eversberg et al. 1998; Markova et al. 2005; Bouret et al. 2005; Fullerton et al. 2006; Oskinova et al. 2006), but also for high mass X-ray binaries (e.g., Torrejón et al. 2015).

Speculatively, the picture of the dip behavior would then be that observations at phase 0 , which sample the region of the stellar wind closer to the donor star, should show a wide spectrum of clump sizes. Observations at phase 0.75 sample regions that are farther away from the wind, where the clump size distribution has changed, probably favoring larger clumps, resulting in less short-term $N_{\mathrm{H}}$ variation. Clearly, however, using only three observations from different orbits and because of the need to perform joint spectroscopy of multiple dips, we do not have sufficient statistics to make a firm statement about the distribution and size of the clumps. Our estimates of $(0.02-0.2) R_{*}$ for the diameter of a clump are in agreement with the high end of the recent 2D simulation results of Sundqvist et al. (2018), however, who have found a typical length scale for clumps of $0.01 R_{*}$ at a distance of two stellar radii from the star. We cannot distinguish between quasi-spherical clumps and, for instance, pancake-shaped clumps as proposed by Oskinova et al. (2012), which would have different optical depths for different lines of sight. Sundqvist et al. (2018) have even found various different clump shapes coexisting in their 2D simulations. Athena with its larger effective area will provide more insight as it will be able to look at single dips.

Acknowledgements. We thank the referee for their very constructive report which improved the paper. The research leading to these results was funded by the Bundesministerium für Wirtschaft und Technologie under grant numbers DLR 50 OR 0701 and DLR 50 OR 1113, by LLNL under Contract DE-AC5207NA27344, and is supported by NASA grants to LLNL. V.G. is supported through the Margarete von Wrangell fellowship by the ESF and the Ministry of Science, Research and the Arts Baden-Württemberg. This research has made use of ISIS functions provided by ECAP/Remeis observatory and MIT (http:// www.sternwarte.uni-erlangen.de/isis/). We thank John E. Davis for providing the slxfig module used for creating the presented plots. We thank Ivica Miškovičová for her initial reduction of the data sets used here.

\section{References}

Bałucińska-Church, M., Church, M. J., Charles, P. A., et al. 2000, MNRAS, 311, 861

Blondin, J. M. 1994, ApJ, 435, 756

Blondin, J. M., \& Woo, J. W. 1995, ApJ, 445, 889

Blondin, J. M., Kallman, T. R., Fryxell, B. A., \& Taam, R. E. 1990, ApJ, 356, 591

Blondin, J. M., Stevens, I. R., \& Kallman, T. R. 1991, ApJ, 371, 684

Bode, M. F., Priedhorsky, W. C., Norwell, G. A., \& Evans, A. 1985, ApJ, 299, 845

Boroson, B., \& Vrtilek, S. D. 2010, ApJ, 710, 197

Bouret, J., Lanz, T., \& Hillier, D. J. 2005, A\&A, 438, 301

Bowyer, S., Byram, E. T., Chubb, T. A., \& Friedman, H. 1965, Ann. Astrophys., 28, 791

Brocksopp, C., Fender, R. P., Larionov, V., et al. 1999, MNRAS, 309, 1063

Caballero-Nieves, S. M., Gies, D. R., Bolton, C. T., et al. 2009, ApJ, 701, 1895

Canizares, C. R., Davis, J. E., Dewey, D., et al. 2005, PASP, 117, 1144

Castor, J. I., Abbott, D. C., Klein, R. I. 1975, ApJ, 195, 157

Dessart, L., \& Owocki, S. P. 2003, A\&A, 406, L1

Dotani, T., Inoue, H., Mitsuda, K., et al. 1997, ApJ, 485, L87

Drake, G. W. 1988, Can. J. Phys., 66, 586

El Mellah, I., Sander, A. A. C., Sundqvist, J. O., \& Keppens, R. 2019, A\&A, 622, A189

Eversberg, T., Lepine, S., \& Moffat, A. F. J. 1998, ApJ, 494, 799

Feldmeier, A., Puls, J., Pauldrach, A. W. A. 1997, A\&A, 322, 878

Feng, Y. X., \& Cui, W. 2002, ApJ, 564, 953

Feng, Y. X., Tennant, A. F., \& Zhang, S. N. 2003, ApJ, 597, 1017

Friend, D. B., \& Castor, J. I. 1982, ApJ, 261, 293

Fullerton, A. W., Massa D. L., \& Prinja, R. K. 2006, ApJ, 637, 1025

Gandhi, P., Rao, A., Johnson, M. A. C., et al. 2019, MNRAS, 485, 2642
Garcia, J. D., \& Mack, J. E. 1965, J. Opt. Soc. Am., 55, 654 Gies, D. R., \& Bolton, C. T. 1986a, ApJ, 304, 371 Gies, D. R., \& Bolton, C. T. 1986b, ApJ, 304, 389 Gies, D. R., Bolton, C. T., Thomson, J. R., et al. 2003, ApJ, 583, 424 Gies, D. R., Bolton, C. T., Blake, R. M., et al. 2008, ApJ, 678, 1237 Grevesse, N., Noels, A., \& Sauval, A. J. 1996, in Cosmic Abundances, eds S. S. Holt \& G. Sonneborn (San Francisco: ASP Conf. Ser.), 99, 117 Grinberg, V., Hell, N., Pottschmidt, K., et al. 2013, A\&A, 554, A88 Grinberg, V., Leutenegger, M. A., Hell, N., et al. 2015, A\&A, 576, A117 Grinberg, V., Hell, N., El Mellah, I., et al. 2017, A\&A, 608, A143

Hanke, M., Wilms, J., Nowak, M. A., et al. 2008, in VII Microquasar Workshop: Microquasars and Beyond. PoS (MQW7), 29

Hanke, M., Wilms, J., Nowak, M. A., et al. 2009, ApJ, 690, 330

Hell, N., Miškovičová, I., Brown, G. V., et al. 2013, Phys. Scr., T156, 014008

Hell, N., Brown, G. V., Wilms, J., et al. 2016, ApJ, 830, 26

Hemphill, P. B., Rothschild, R. E., Markowitz, A., et al. 2014, ApJ, 792, 14

Herrero, A., Kudritzki, R. P., Gabler, R., et al. 1995, A\&A, 297, 556

Houck, J. C. 2002, in High Resolution X-ray Spectroscopy with XMM-Newton and Chandra, Mullard Space Science Laboratory, Holmbury St Mary

Houck, J. C., \& Denicola, L. A. 2000, in Astronomical Data Analysis Software and Systems IX, eds. N. Manset, C. Veillet, \& D. Crabtree, ASP Conf. Ser., 216, 591

Kallman, T., \& Bautista, M. 2001, ApJS, 133, 221

Kitamoto, S., Miyamoto, S., Tanaka, Y., et al. 1984, PASJ, 36, 731

Krolik, J. H., McKee, C. F., \& Tarter, C. B. 1981, ApJ, 249, 422

Li, K. F., \& Clark, G. W. 1974, ApJ, 191, L27

Manousakis, A., \& Walter, R. 2011, A\&A, 526, A62

Manousakis, A., \& Walter, R. 2015, A\&A, 575, A58

Markova, N., Puls, J., Scuderi, S., \& Markov, H. 2005, A\&A, 440, 1133

Martínez-Núñez, S., Kretschmar, P., Bozzo, E., et al. 2017, Space Sci. Rev., 212, 59

Mason, K. O., Hawkins, F. J., Sanford, P. W., et al. 1974, ApJ, 192, L65

Miller, J. M., Fabian, A. C., Wijnands, R., et al. 2002, ApJ, 578, 348

Miškovičová, I., Hanke, M., Wilms, J., et al. 2011, Acta Polytech., 51, 4

Miškovičová, I., Hell, N., Hanke, M., et al. 2016, A\&A, 590, A114

Morton, D. C. 1967, ApJ, 150, 535

Muijres, L. E., Vink, J. S., de Koter, A., et al. 2012, A\&A, 537, A37

Murdin, P., \& Webster, B. L. 1971, Nature, 233, 110

Noble, M. S., \& Nowak, M. A. 2008, PASP, 120, 821

Nowak, M. A., Vaughan, B. A., Wilms, J., et al. 1999, ApJ, 510, 874

Nowak, M. A., Hanke, M., Trowbridge, S. N., et al. 2011, ApJ, 728, 13

Orosz, J. A., McClintock, J. E., Aufdenberg, J. P., et al. 2011, ApJ, 742, 84

Oskinova, L. M., Feldmeier, A., \& Hamann, W. 2006, MNRAS, 372, 313

Oskinova, L. M., Feldmeier, A., \& Kretschmar, P. 2012, MNRAS, 421, 2820

Owocki, S. P., \& Rybicki, G. B. 1984, ApJ, 284, 337

Owocki, S. P., Castor, J. I., \& Rybicki, G. B. 1988, ApJ, 335, 914

Palmeri, P., Quinet, P., Mendoza, C., et al. 2008, ApJS, 177, 408

Parker, M. L., Tomsick, J. A., Miller, J. M., et al. 2015, ApJ, 808, 9

Parsignault, D. R., Epstein, A., Grindlay, J., et al. 1976, Ap\&SS, 42, 175

Pepe, C., Vila, G. S., \& Romero, G. E. 2015, A\&A, 584, A95

Porquet, D., Dubau, J., \& Grosso, N. 2010, Space Sci. Rev., 157, 103

Poutanen, J., Zdziarski, A. A., Ibragimov, A. 2008, MNRAS, 389, 1427

Pravdo, S. H., White, N. E., Becker, R. H., et al. 1980, ApJ, 237, L71

Puls, J., Markova, N., Scuderi, S., et al. 2006, A\&A, 454, 625

Puls, J., Vink, J. S., \& Najarro, F. 2008, A\&ARv, 16, 209

Rahoui, F., Lee, J. C., Heinz, S., et al. 2011, ApJ, 736, 63

Reid, M. J., McClintock, J. E., Narayan, R., et al. 2011, ApJ, 742, 83

Remillard, R. A., \& Canizares, C. R. 1984, ApJ, 278, 761

Sako, M., Liedahl, D. A., Kahn, S. M., \& Paerels, F. 1999, ApJ, 525, 921

Schulz, N. S., Cui, W., Canizares, C. R., et al. 2002a, ApJ, 565, 1141

Schulz, N. S., Canizares, C. R., Lee, J. C., \& Sako, M. 2002b, ApJ, 564, L21

Sundqvist, J. O., \& Owocki, S. P. 2013, MNRAS, 428, 1837

Sundqvist, J. O., Owocki, S. P., Cohen, D. H., et al. 2012, MNRAS, 420, 1553

Sundqvist, J. O., Owocki, S. P., Puls, J. 2018, A\&A, 611, A17

Tomsick, J. A., Nowak, M. A., Parker, M., et al. 2014, ApJ, 780, 78

Torrejón, J. M., Schulz, N. S., Nowak, M. A., et al. 2015, ApJ, 810, 102

Verner, D. A., Verner, E. M., \& Ferland, G. J. 1996, At. Data Nucl. Data Tables, 64,1

Vrtilek, S. D., Boroson, B. S., Hunacek, A., et al. 2008, ApJ, 678, 1248

Walborn, N. R. 1973, ApJ, 179, p.L123

Watanabe, S., Sako, M. Ishida, M., et al. 2006, ApJ, 651, 421

Webster, B. L., \& Murdin, P. 1972, Nature, 235, 37

Wen, L., Cui, W., Levine, A. M., \& Bradt, H. V. 1999, ApJ, 525, 968

Wilms, J., Allen, A., \& McCray, R. 2000, ApJ, 542, 914

Wilms, J., Nowak, M. A., Pottschmidt, K., et al. 2006, A\&A, 447, 245

Wojdowski, P. S., Liedahl, D. A., Sako, M., et al. 2003, ApJ, 582, 959

Xiang, J., Lee, J. C., Nowak, M. A., \& Wilms, J. 2011, ApJ, 738, 78

Zhang, S. N., Cui, W., Harmon, B. A., et al. 1997, ApJ, 477, L95 